UNITUE-THEP-6/98

nucl-th/9805054

Draft : June 19, 2018

\title{
Octet and Decuplet Baryons in a Covariant and Confining Diquark-Quark Model ${ }^{\dagger}$
}

\author{
M. Oettel*, G. Hellstern ${ }^{\$}$, R. Alkofer ${ }^{\#}$ and H. Reinhardt \\ Institute for Theoretical Physics, \\ Tübingen University, \\ Auf der Morgenstelle 14, \\ D-72076 Tübingen, Germany
}

\begin{abstract}
The baryon octet and decuplet masses and Bethe-Salpeter vertex and wave functions are calculated in the ladder approximation to the quark exchange between a scalar or axialvector diquark and a constituent quark. These functions reflecting full Lorentz covariance are given in terms of an expansion in Gegenbauer polynomials. In the rest frame of the baryon, a complete partial wave decomposition of the Bethe-Salpeter wave function is performed. The confinement of quarks and diquarks is implemented via a parametrisation of the corresponding propagators. We also discuss some aspects of the momentum routing in the ladder approximation to the Bethe-Salpeter equation. Numerical results for the octet and decuplet masses with broken flavour $S U(3)$ in the conserved isospin limit are presented.
\end{abstract}

Keywords: diquarks, Bethe-Salpeter equation, baryon masses

PACS: 11.10.st, 12.39.Ki, 12.40.yx, 14.20.Dh. 14.20Jn

$\dagger$ Supported by BMBF under contract 06TU888, DFG under contract We 1254/4-1 and Graduiertenkolleg

"Hadronen und Kerne" (DFG Mu705/3).

* E-mail: oettel@pthp3.tphys.physik.uni-tuebingen.de

\$Address after June $1^{\text {st }}$, 1998: Deutsche Bank, Frankfurt.

\# E-mail: Reinhard.Alkofer@uni-tuebingen.de 


\section{Introduction}

In a recent paper [1] we presented numerical results for the nucleon form factors in a fully Lorentz covariant model based on the idea that baryons may be viewed as bound states of confined constituent diquarks and quarks interacting via quark exchange. The confinement of the constituents is hereby effectively parametrised. The poles of the corresponding propagators are removed by some modifying multiplicative factors which, however, possess an essential singularity at time-like infinite momentum. The physical picture behind this baryon model is very natural: Diquarks are allowed to "decay" into two quarks, one of them recombines with the third quark and forms another diquark. The hope is, that for physics relevant at intermediate momentum transfers, most of the complicated structure of the baryon may be efficaciously described by assuming strong correlations in the quark-quark channel. Thus the notion of diquarks parametrises to some extend unknown non-perturbative physics within baryons. In the last years diquarks have not only been used in non-perturbative calculations but also in the description of inelastic leptonnucleon scattering, see refs. [2, [3] where various applications of diquarks are extensively discussed. Very recently diquark masses have been estimated from lattice measurements [1].

The main purpose of our investigations is to formulate a baryon model applicable to the intermediate energy region. This is mainly motivated with the advent of a new generation of continuous beam facilities like CEBAF at TJNAF, MAMI, ELSA, COSY etc. which are designed to explore an intermediate region lying between the non-perturbative lowenergy and the perturbative high-energy regime of QCD. These facilities explore various hadron observables to a very high precision. The different existing hadron models are to be judged by their ability to predict and to explain these observables in the near future. While there exist many models capable to describe pion properties which are strongly dictated by chiral symmetry, the detailed structure of all other mesons are still unclear. Although quirte a few baryon models, see e.g. [5, 6, 7, 8, 9, 10, 11], were developed in the last forty years, a unified description of baryons within a covariant field theoretical approach, and with quarks and gluons as the fundamental degrees of freedom, is still missing. Besides being covariant, such a description should include chiral symmetry in its spontaneously broken phase and confinement. A covariant model with the correct symmetry pattern but without confinement is the Nambu-Jona-Lasinio (NJL) model [12, 13] where quarks as fundamental fermion fields interact locally, for recent reviews see [14, 15, 16]. There, baryons appear either as non-topological solitons [17, 18, 19] or as bound states of a quark and a diquark [20, 21, 22, 23, 24, 25, 26]. A hybrid formalism combining the soliton with the diquark picture has been developed in ref. [27], where it turned out, that the 
soliton background contributes as much to the total binding energy of the nucleon as the direct coupling between two quarks and between quark and diquark. In the Global Colour Model, a non-local extension of the NJL model, there also exist preliminary studies of nucleons as diquark-quark bound states, see e.g. [28] and references therein.

In this paper we extend the investigations reported in ref. [1]. There we solved the Bethe-Salpeter equation for nucleons in ladder approximation but restricted ourselves to scalar diquarks. Employing the solution of the Bethe-Salpeter equation, i.e. the nucleon vertex functions, we calculated various form factors like the electromagnetic, the weak and the pionic form factor of the nucleon. Despite the fact that this work is a promising starting point for further investigations, some results, especially for the magnetic moments, signalled that the axialvector diquark channel is necessary for a realistic description of baryons as bound states of quarks and diquarks. Here we therefore include the axialvector diquark channel into the nucleon Bethe-Salpeter equation. We also present results for baryons with spin $3 / 2$.

The paper is organised as follows: In the next section the covariant and confining diquark-quark model, as defined in ref. [1], is briefly reviewed. By modifying the propagators of quarks and diquarks an effective modelling of confinement enters our model. In section 3 the Bethe-Salpeter equations for spin- $1 / 2$ and spin-3/2 baryons, determining their masses and wave functions, are discussed. With an appropriate three spinor basis we then construct covariant ansätze for the wave functions which are suitable for the numerical solution of the homogeneous integral equations. Section 1 is devoted to a discussion of the subtleties concerning the momentum routing in the Bethe-Salpeter equation, associated with the ladder approximation. The numerical method is shortly described in section 5, and our results for the baryon masses and wave functions are described in section 6. In the last section we finally conclude and give an outlook. Some technical issues are deferred to three appendices.

\section{The Covariant and Confining Diquark-Quark Model}

Here we briefly recapitulate the definition of the covariant and confining diquark-quark model as given in ref. [四. Such a model, although without confinement has been also considered in [29, 30]. Since the solution of a rigorous relativistic three body equation, a Faddeev equation [31, is still missing in field theory we follow the path of using diquarks as effective degrees of freedom within baryons. They serve as an efficient tool to parametrise some of the unknown non-perturbative features of the baryon wave function. As stated 
in the introduction such approaches have been successful in the past to describe baryons in NJL-type models [20, 21, 22, 23, 24, 25, 26]. A three-dimensional reduction of the fully covariant Bethe-Salpeter model discussed here, can be found in ref. [32], where the author solved the bound state equations in the Salpeter approximation which ignores all retardation effects.

Having in mind the diquark-quark description arising within the hadronized NJL model [20], some basic structures are fixed: To build up a colourless baryon out of a diquark and a quark being in the fundamental representation of the colour group $\mathrm{SU}(3)_{\mathrm{C}}$, diquarks necessarily live in the colour anti-triplet channel. Furthermore, in order to fulfil the Pauli principle, scalar diquarks couple via the antisymmetric generators of the flavour group, $t_{\mathcal{A}}^{a}=\left\{\rho^{a=1 . .3}\right\}$, axialvector diquarks via the symmetric generators $t_{\mathcal{S}}^{a}=\left\{\rho^{a=4 . .9}\right\}$, respectively [20]. Our conventions for these flavour matrices $\rho^{a}$ are given in appendix A. In the following, however, we deviate from the NJL model as described in [16]. Quarks and diquarks are treated as elementary but confined particles, see below, whose interaction, quark exchange, gives rise to quark-diquark correlations strong enough to bind these fields to a baryon.

This can be formalised with the Lagrangian

$$
\begin{aligned}
\mathcal{L}= & \bar{q}_{A}(x)\left(i \gamma^{\mu} \partial_{\mu}-\mathbf{m}_{q}\right) f\left(-\partial^{2} / \mathbf{m}_{q}^{2}\right) q_{A}(x) \\
& +\Delta_{A}^{\dagger}(x)\left(-\partial_{\mu} \partial^{\mu}-\mathbf{m}_{0^{+}}^{2}\right) f\left(-\partial^{2} / \mathbf{m}_{0^{+}}^{2}\right) \Delta_{A}(x) \\
& -\frac{1}{4} F_{\mu \nu}^{\dagger}(x) f\left(-\partial^{2} / \mathbf{m}_{1^{+}}^{2}\right) F^{\mu \nu}(x)+\frac{1}{2} \mathbf{m}_{1^{+}}^{2} \Delta_{A a \mu}^{\dagger}(x) f\left(-\partial^{2} / \mathbf{m}_{1^{+}}^{2}\right) \Delta_{A a}^{\mu}(x) \\
& +\epsilon^{A B C}\left(g_{s} q_{C}^{T}(x) C i \gamma^{5} t_{\mathcal{A}}^{a} q_{B}(x) \Delta_{a A}^{*}(x)+g_{s}^{*} \Delta_{a A}(x) \bar{q}_{B}(x) i \gamma^{5} C t_{\mathcal{A}}^{\dagger a} \bar{q}_{C}^{T}(x)\right) \\
& +\epsilon^{A B C}\left(g_{a} q_{C}^{T}(x) C i \gamma^{\mu} t_{\mathcal{S}}^{a} q_{B}(x) \Delta_{A a \mu}^{*}(x)-g_{a}^{*} \Delta_{A a \mu}(x) \bar{q}_{B}(x) t_{\mathcal{S}}^{\dagger a} i \gamma^{\mu} C \bar{q}_{C}^{T}(x)\right) .
\end{aligned}
$$

The quark field is denoted by $q(x)$, the scalar diquark field by $\Delta(x)$ and the axialvector diquark field by $\Delta^{\mu}(x)$. Their masses are given by the matrices in flavour space $\mathbf{m}_{q}, \mathbf{m}_{0^{+}}$ and $\mathbf{m}_{1^{+}}$, respectively. For unbroken flavour symmetry they reduce to $\mathbf{m}_{q}=\operatorname{diag}_{3}\left(m_{q}\right)$, $\mathbf{m}_{0^{+}}=\operatorname{diag}_{3}\left(m_{0^{+}}\right)$and $\mathbf{m}_{1^{+}}=\operatorname{diag}_{6}\left(m_{1^{+}}\right)$. In eq. (11), capital subscripts denote colour quantum numbers. In the kinetic part of the $1^{+}$diquark the non-abelian field strength tensor $F^{\mu \nu}=\partial^{\mu} \Delta^{\nu}-\partial^{\nu} \Delta^{\mu}+\left[\Delta^{\mu}, \Delta^{\nu}\right]$ appears $\left(\Delta_{\mu}=\Delta_{a \mu} t_{\mathcal{S}}^{a}\right)$. The associated selfinteractions, however, will not be taken into account. The coupling strengths, of Yukawa type, between two quarks and the scalar or axialvector diquarks are given by $g_{s}$ or $g_{a}$, respectively. In order to take an extended diquark into account, the point-like couplings will be supplemented with momentum dependent factors to be defined later.

An essential ingredient entering our model is the effective parametrisation of confinement. This is realised by modifying the kinetic terms of the constituents in the Lagrangian 
(11). Going to Euclidean円 momentum space and using

$$
f^{-1}(x)=1-e^{-d(1+x)}
$$

the model quark and diquark propagators, being diagonal in colour and flavour space, are given by

$$
\begin{aligned}
S(p) & =\frac{i p-m_{q}}{p^{2}+m_{q}^{2}}\left(1-e^{-d\left(p^{2}+m_{q}^{2}\right) / m_{q}^{2}}\right), \\
D(p) & =-\frac{1}{p^{2}+m_{0^{+}}^{2}}\left(1-e^{-d\left(p^{2}+m_{0^{+}}^{2}\right) / m_{0^{+}}^{2}}\right), \\
D^{\mu \nu}(p) & =-\frac{\left(\delta^{\mu \nu}+p^{\mu} p^{\nu} / m_{1^{+}}^{2}\right)}{p^{2}+m_{1^{+}}^{2}}\left(1-e^{-d\left(p^{2}+m_{1^{+}}^{2}\right) / m_{1^{+}}^{2}}\right) .
\end{aligned}
$$

Due to the numerators, the mass poles of the propagators are effectively screened[? The strength of the screening is described by the parameter $d$. Whereas the analytic behaviour of the quark propagator (3) is similar to the ones obtained from Dyson-Schwinger studies of QCD (details about this approach can be found in ref.[33]), the justification for using confined diquarks is somewhat more involved. As stated above, diquarks are not colour singlets and should therefore be confined by the fundamental interaction of QCD. Nevertheless most diquark models [34, 35, 36] which describe them as bound states of two quarks predict diquarks to be observable particles. Recent studies, however, which investigated the system of the quark Dyson-Schwinger equation and the diquark BetheSalpeter equation beyond the usually employed rainbow-ladder approximation, were able to explain why diquarks do not appear in the observable particle spectrum. This mechanism works in the Munczek-Nemirovsky model [37], as has been shown in ref. [38], as well as in an extended NJL model [39]. Since both models assume a simplified but quite different quark-quark interaction, one may conjecture that the same also holds true implementing a realistic interaction. For these reasons we use the notion of confined diquarks. In our approach we therefore use the propagators defined in eqs. (4, 5) which do not allow observable diquarks. In the following it will be seen, that working with confined quarks and diquarks leads to the absence of an unphysical quark-diquark threshold for baryons.

Additionally we introduce the diagonal approximation to the axialvector diquark propagator by omitting the $p^{\mu} p^{\nu} / m_{1+}^{2}$ term,

$$
D^{\mu \nu}(p)=-\frac{\delta^{\mu \nu}}{p^{2}+m_{1^{+}}^{2}} f^{-1}\left(\frac{p^{2}}{m_{1^{+}}^{2}}\right) .
$$

It is well-known that the full Proca propagator may cause spurious ultraviolet problems. These vanish in the special, unitary gauge and have no physical consequences. As such a

\footnotetext{
${ }^{1}$ We use an Euclidean space formulation with $\left\{\gamma_{\mu}, \gamma_{\nu}\right\}=2 \delta_{\mu \nu}, \gamma_{\mu}^{\dagger}=\gamma_{\mu}$ and $p q=\sum_{\mu=1}^{4} p_{\mu} q_{\mu}$.

${ }^{2}$ With $f \equiv 1$ we refer to the propagators as tree level propagators.
} 
treatment is, however, beyond the scope of our purely phenomenological investigation we have chosen to avoid this problem by using a diagonal approximation to the propagator. Its validity is examined more closely in appendix B.

\section{Bethe-Salpeter Equation for Octet and Decuplet Baryons in the Flavour-Symmetric Case}

Using the Lagrangian given in eq. (II) we obtain the ladder Bethe-Salpeter equation for octet and decuplet baryons,

$$
\begin{aligned}
\left(\begin{array}{c}
\Psi_{8}(p ; P) \\
\Psi_{8}^{\nu}(p ; P)
\end{array}\right)= & -\left|g_{s}\right|^{2}\left(\begin{array}{cc}
D\left(p_{b}\right) & 0 \\
0 & D^{\nu \mu}\left(p_{b}\right)
\end{array}\right) S\left(p_{a}\right) \\
& \times \int \frac{d^{4} p^{\prime}}{(2 \pi)^{4}}\left(\begin{array}{cc}
\gamma_{5} \tilde{S}(-q) \gamma_{5} & -\sqrt{3} \frac{g_{a}}{g_{s}} \gamma^{\mu^{\prime}} \tilde{S}(-q) \gamma_{5} \\
-\sqrt{3} \frac{g_{a}^{*}}{g_{s}^{*}} \gamma_{5} \tilde{S}(-q) \gamma^{\mu} & -\frac{\left|g_{a}\right|^{2}}{\left|g_{s}\right|^{2}} \gamma^{\mu^{\prime}} \tilde{S}(-q) \gamma^{\mu}
\end{array}\right)\left(\begin{array}{c}
\Psi_{8}\left(p^{\prime} ; P\right) \\
\Psi_{8}^{\mu^{\prime}}\left(p^{\prime} ; P\right)
\end{array}\right) \\
\Psi_{10}^{\nu \rho}(p ; P)= & -2\left|g_{a}\right|^{2} S\left(p_{a}\right) D^{\nu \mu}\left(p_{b}\right) \int \frac{d^{4} p^{\prime}}{(2 \pi)^{4}} \gamma^{\lambda} \tilde{S}(-q) \gamma^{\mu} \Psi_{10}^{\lambda \rho}\left(p^{\prime} ; P\right)
\end{aligned}
$$

In this formulation, the Bethe-Salpeter equation involves the matrix-valued Bethe-Salpeter wave functions of octet baryons $\Psi_{8}(p ; P)$ and $\Psi_{8}^{\nu}(p ; P)$ and decuplet baryons $\Psi_{10}^{\lambda \nu}(p ; P)$, respectively, which are projected on positive parity and spin $1 / 2$ or spin $3 / 2$ (see the following subsection for their construction). Their flavour part is given by pure octet and decuplet states in $S U(3)_{\text {flavour }}$. They depend on the total momentum $P$ of the bound state and on the relative momentum $p^{\prime}$ or $p$ between the two constituents. Mathematically, the Bethe-Salpeter equations are equivalent to coupled homogeneous integral equations. The numerical method for its solution is presented in sect. 57, see also [40].

Although the Lagrangian (1) describes a renormalisable diquark-quark theory, at leat at one-loop level, the Bethe-Salpeter equations in ladder approximation are formally divergent in the ultraviolet. The divergence, of course, should then be cured by the wave functions. However, to crudely take into account the extended nature of diquarks we work, as in ref. [30, 1], with a finite interaction in momentum space and modify the propagator of the exchanged quark according to

$$
S(q) \rightarrow \tilde{S}(q)=S(q)\left(\frac{\Lambda^{2}}{q^{2}+\Lambda^{2}}\right)
$$

This corresponds to a monopole-type form factor. As a consequence, this also removes all formal ultraviolet divergencies. Note that we have absorbed the charge conjugation matrix $C$ appearing in the Lagrangian (1) using the identity $C^{-1} \tilde{S}^{T}(q) C=\tilde{S}(-q)$.

Before actually solving the integral equations it is appropriate to find a suitable basis for the wave functions appearing in eqs. (77,8). 


\subsection{Relativistic Three Quark States and their Wave Functions}

When constructing a baryon out of three quarks, its wave function is formally described by a spinor of rank three, $\psi_{\alpha \beta \gamma}(\alpha, \beta, \gamma=1 \ldots 4)$. For octet baryons, a convenient basis for this multi-spinor can be found by expanding the direct product of the spinors describing quarks of flavour $b$ and $c$ with spinor indices $\beta$ and $\gamma$ into the complete set of Dirac matrices and taking the direct product with a spinor basis of quark $a$ [1]:

$$
\psi_{\alpha \beta \gamma}^{8}=(\Gamma u)_{\alpha}\left(\phi C \gamma_{5}\right)_{\beta \gamma} .
$$

$C$ denotes charge conjugation and $u=\left\{\left(\begin{array}{c}\chi^{+} \\ 0\end{array}\right),\left(\begin{array}{c}\chi^{-} \\ 0\end{array}\right)\right\}$ is a basis of positive energy Dirac spinors in the rest frame describing fermions with spin up $(+)$ and spin down $(-)$. In this representation $\Gamma$ and $\phi$ are Dirac matrices to be expanded in the complete set $\left\{\mathbf{1}, \gamma_{5}, \gamma^{\mu}, \gamma_{5} \gamma^{\mu}, \sigma^{\mu \nu}\right\}$.

Under a Lorentz transformation $S(\Lambda)$, this wave function transforms according to

$$
\psi_{\alpha \beta \gamma}^{8}=\left(S \Gamma\left(\Lambda^{-1} P, \Lambda^{-1} p\right) S^{-1}\right)_{\alpha \alpha^{\prime}}(S u)_{\alpha^{\prime}}\left(S \phi S^{-1} C \gamma_{5}\right)_{\beta \gamma} .
$$

The parity transformation is given by

$$
\psi_{\alpha \beta \gamma}^{8}=\pi\left(\gamma^{4} \Gamma\left(\tilde{\gamma}^{\mu}, \tilde{P}, \tilde{p}\right) u\right)_{\alpha}\left(\gamma^{4} \phi\left(\tilde{\gamma}^{\mu}, \tilde{P}, \tilde{p}\right) \gamma^{4} C \gamma_{5}\right)_{\beta \gamma},
$$

where $\pi$ is the intrinsic parity of the baryon and $\tilde{P}=\left(-\mathbf{P}, P^{4}\right), \tilde{p}=\left(-\mathbf{p}, p^{4}\right), \tilde{\gamma}^{\mu}=$ $\left(-\gamma, \gamma^{4}\right)$.

To ensure total spin $1 / 2$ of the wave function, the free Lorentz indices in (10) are to be contracted with the independent momenta involved and each covariant will be multiplied by a scalar function.

In the diquark-quark model, only the two choices for $\phi=\left\{\mathbf{1}, \gamma^{\mu} \gamma_{5}\right\}$ are taken into account which describe the scalar and axialvector diquark. The covariants which lead to positive parity of spin-1/2 octet states, $(\Gamma u)_{\alpha}$, can be grouped according to scalar and axialvector diquark states with the spinor indices $\beta$ and $\gamma$, see table 1, second row. As described above, the free Lorentz indices in these covariants are contracted with the momenta $P$ and $p$ and are then to be multiplied with scalar functions. No further symmetrisation of the wave function is necessary as this will be provided by the quark exchange.

Using this classification scheme, the octet wave function can be denoted by

$$
\psi_{\alpha \beta \gamma}^{8}=\sum_{i=1}^{4}\left(Z_{i}^{S} \Gamma_{i}^{S} u\right)_{\alpha}\left(C \gamma_{5}\right)_{\beta \gamma}+\sum_{i=5}^{16}\left(Z_{i}^{A} \Gamma_{i}^{A \mu} u\right)_{\alpha}\left(\gamma_{\mu} C\right)_{\beta \gamma} .
$$

Each of the four covariants $\Gamma_{S}^{i}$ which describe the scalar diquark part in the octet baryon wave function is multiplied by a scalar function $Z_{i}^{S}$ and likewise there are twelve scalar functions $Z_{i}^{A}$ multiplying the axialvector diquark covariants $\Gamma_{i}^{A \mu}$. 


\begin{tabular}{|c|c|c|}
\hline & Scalar Diquark - $\left(C \gamma_{5}\right)_{\beta \gamma}$ & Axialvector Diquark - $\left(\gamma_{\mu} \gamma_{5} C \gamma_{5}\right)_{\beta \gamma}$ \\
\hline Octet & $\left(\Gamma_{i}^{S} u\right)_{\alpha}$ & $\left(\Gamma_{i}^{A \mu} u\right)_{\alpha}$ \\
\hline Decuplet & & $\begin{array}{l}\left(\Gamma_{i}^{S} u^{\mu}\right)_{\alpha} \\
\left(\gamma_{5} \Gamma_{i}^{A \mu}\left[p_{T}^{\nu} u_{\nu}\right]\right)_{\alpha}\end{array}$ \\
\hline $\bar{\Gamma} \Gamma_{i}^{S} \in$ & \multicolumn{2}{|l|}{$\mathbf{1}, P p, \not p, P_{\mu} \sigma^{\mu \nu} p_{\nu}$} \\
\hline$\Gamma_{i}^{A \mu} \in$ & \multicolumn{2}{|c|}{$\begin{array}{l}\left\{P^{\mu} \gamma_{5}, p^{\mu} \gamma_{5}, \gamma^{\mu} \gamma_{5}, P^{\mu} \gamma_{5} P, P^{\mu} \gamma_{5} \not p\right. \\
p^{\mu} \gamma_{5} \not P, p^{\mu} \gamma_{5} p, P_{\nu} \gamma_{5} \sigma^{\nu \mu}, p_{\nu} \gamma_{5} \sigma^{\nu \mu} \\
\left.P^{\mu} \gamma_{5} P_{\nu} \sigma^{\nu \rho} p_{\rho}, p^{\mu} \gamma_{5} P_{\nu} \sigma^{\nu \rho} p_{\rho}, \gamma^{\mu} \gamma_{5} P_{\nu} \sigma^{\nu \rho} p_{\rho}\right\}\end{array}$} \\
\hline
\end{tabular}

Table 1: The Lorentz covariants leading to baryons with positive parity. They are grouped according to their diquark content.

A further reduction of this ansatz by a projection to positive energies is very convenient. From the expression (13) for the octet wave function we consider only the part with spinor index $\alpha$, multiply it with the adjoint spinor $\bar{u}(P, s)$ and sum over the spins. This leads to a wave function which is, by construction, an eigenfunction of the positive-energy projector $\Lambda^{+}=(1+\hat{P})$. Thus we are led to the following relations between the wave functions of eq. (13) and the corresponding functions in the Bethe-Salpeter equation (7):

$$
\left(\begin{array}{c}
Z_{i}^{S} \Gamma_{i}^{S} \\
Z_{i}^{A} \Gamma_{i}^{A \mu}
\end{array}\right) \Lambda^{+}=\left(\begin{array}{c}
S_{i} \mathcal{S}_{i} \\
A_{i} \mathcal{A}_{i}^{\mu}
\end{array}\right)=\left(\begin{array}{c}
S_{i} \mathcal{S}_{i} \\
A_{i} \mathcal{A}_{i}^{\mu}
\end{array}\right) \Lambda^{+}=\left(\begin{array}{c}
\Psi_{8}(p ; P) \\
\Psi_{8}^{\mu}(p ; P)
\end{array}\right)
$$

The independent covariants $\mathcal{S}_{i}$ and $\mathcal{A}_{i}^{\mu}$ are required to be eigenfunctions of $\Lambda^{+}$which reduces the number of independent scalar functions from sixteen to eight which are now denoted by $S_{i},(\mathrm{i}=1,2)$ and $A_{i},(\mathrm{i}=1 \ldots 6)$. A convenient representation of these covariants suitable for our numerical procedure and for further applications is given by

$$
\begin{aligned}
& \mathcal{S}_{i}=\left\{\begin{array}{l}
\mathcal{S}_{1}=\Lambda^{+} \\
\mathcal{S}_{2}=-\frac{i}{p} p_{T} \Lambda^{+}
\end{array}\right. \\
& \mathcal{A}_{i}^{\mu}=\left\{\begin{array}{l}
\mathcal{A}_{1}^{\mu}=-\frac{i}{p} \hat{P}^{\mu} \gamma_{5} p_{T} \Lambda^{+} \\
\mathcal{A}_{2}^{\mu}=\hat{P}^{\mu} \gamma_{5} \Lambda^{+} \\
\mathcal{A}_{3}^{\mu}=\hat{p}_{T}^{\mu} \gamma_{5} p_{T} \Lambda^{+} \\
\mathcal{A}_{4}^{\mu}=\frac{i}{p} p_{T}^{\mu} \gamma_{5} \Lambda^{+} \\
\mathcal{A}_{5}^{\mu}=\gamma_{5} \gamma_{T}^{\mu} \Lambda^{+}-\mathcal{A}_{3}^{\mu} \\
\mathcal{A}_{6}^{\mu}=\frac{i}{p} \gamma_{5} \gamma_{T}^{\mu} p_{T} \Lambda^{+}-\mathcal{A}_{4}^{\mu},
\end{array}\right.
\end{aligned}
$$

${ }^{3}$ With a hat we denote normalised four vectors, e.g. $\hat{P} \cdot \hat{P}=1$. In the Euclidean rest frame $P=(\mathbf{0}, i M)$ this explicitly reads $\hat{P}=P / i M$. Note that all relative momenta $\left(p, p^{\prime}\right)$ are real in Euclidean space as they are only needed for spacelike values. 
where $\gamma_{T}^{\mu}=\gamma^{\mu}-\hat{P}^{\mu} \hat{P}$. Note that the indices have been chosen such that matrices with odd indices $i=\{1,3,5\}$ are eigenfunctions to $P P-i M$ whereas the ones with even indices $i=\{2,4,6\}$ are eigenfunctions to $P+i M$, with eigenvalue 0 in both cases.

In the rest frame of the bound state, $P=(\mathbf{0}, i M)$, the ansatz for the matrix valued nucleon wave function which we used for further numerical processing reads

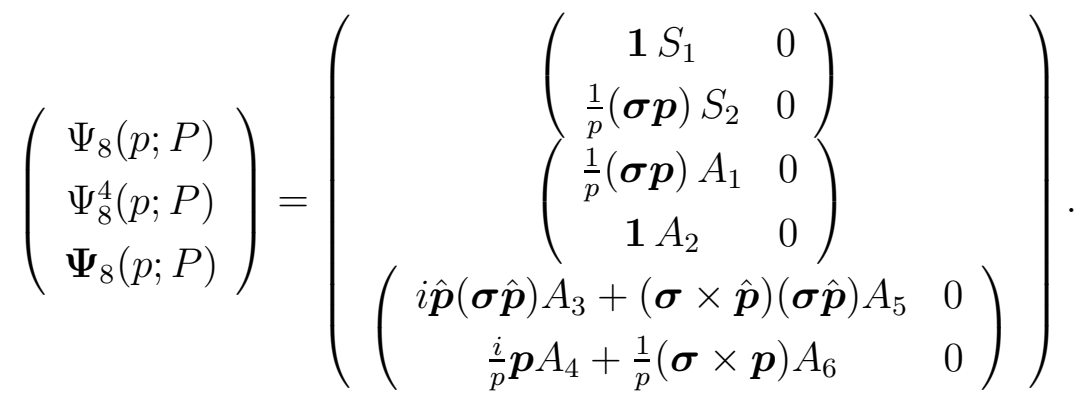

The scalar quantities $S_{i}$ and $A_{i}$ depend on $p=\sqrt{p_{\mu} p_{\mu}}$ and $z=\cos \psi=\hat{P} \cdot \hat{p}$ for each value of the bound state mass. As expected from the properties of $\mathcal{S}_{i}$ and $\mathcal{A}_{i}^{\mu}$ (see eqs. (15) and (16)), upper components have odd indices, lower components have even indices.

The strategy shown above for the octet baryon can also be applied to decuplet baryons which have spin 3/2. Projection of the tri-spinor wave function $\psi_{\alpha \beta \gamma}$ onto total spin $3 / 2$ may be achieved by expanding the piece associated with quark $a$ in terms of RaritaSchwinger spinors $u_{\mu}$ :

$$
\psi_{\alpha \beta \gamma}^{10}=\left(\Gamma u_{\mu}\right)_{\alpha}(\phi C)_{\beta \gamma}
$$

$\Gamma$ is chosen such that all Lorentz indices are contracted with the momenta $P$ and $p$. Note that the Rarita-Schwinger constraints demand $u_{\mu} \gamma^{\mu}=u_{\mu} P^{\mu}=0$ and that in the diquark-quark model the diquark part of decuplet states is made of axialvecor diquarks only, $\phi=C \gamma^{\nu}$.

The covariants $\Gamma$ which are left for decuplet states after applying the above restrictions and the positive parity constraint can be found in table 1, third row. The covariants for octet and decuplet states are closely related. Due to the Rarita-Schwinger constraints the Lorentz index of $u_{\mu}$ must be contracted either with the Lorentz index of the axialvector diquark $\left(\gamma^{\mu} C\right)$ or with the transversal relative momentum $p_{T}^{\mu}=p^{\mu}-\hat{P}^{\mu}(p \cdot \hat{P})$. For the first choice, we have the same four covariants as for the octet state with scalar diquark correlations. For the second choice, we obtain the twelve covariants as for the octet state with axialvector diquark correlations, however, multiplied by $\gamma_{5}$ to ensure positive parity. 
The decuplet wave functions reads now:

$$
\begin{aligned}
\psi_{\alpha \beta \gamma}^{10} & =\left(\Upsilon_{10}^{\mu \nu} u^{\nu}\right)_{\alpha}\left(\gamma_{\mu} C\right)_{\beta \gamma} \\
& =\sum_{i=1}^{4}\left(Z_{i}^{D} \Gamma_{i}^{S} \delta^{\mu \nu} u^{\nu}\right)_{\alpha}\left(\gamma_{\mu} C\right)_{\beta \gamma}+\sum_{i=5}^{16}\left(Z_{i}^{D} \gamma_{5} \Gamma_{i}^{A \mu} \hat{p}_{T}^{\nu} u^{\nu}\right)_{\alpha}\left(\gamma_{\mu} C\right)_{\beta \gamma}
\end{aligned}
$$

where the $Z_{i}^{D}$ denote the sixteen scalar functions for the decuplet state.

Taking from eq. (19) only the part with spinor index $\alpha$ and projecting it to positive energies leads to

$$
\sum_{s=-3 / 2}^{+3 / 2} \Upsilon_{10}^{\mu \nu}(p ; P) u^{\nu}(P, s) \bar{u}^{\lambda}(P, s)=\Upsilon_{10}^{\mu \nu}(p ; P) \mathbf{P}^{\nu \lambda}=\Psi_{10}^{\mu \nu}(p ; P)=\Psi_{10}^{\mu \lambda}(p ; P) \mathbf{P}^{\lambda \nu}
$$

Therefore the projected wave function (which besides being a $4 \times 4$-matrix has tensor character) is determined by the condition

$$
\Psi_{10}^{\mu \nu}(p ; P)=\Psi_{10}^{\mu \lambda}(p ; P) \mathbf{P}^{\lambda \nu},
$$

which requires $\Psi_{10}^{\mu \nu}$ to be an eigenfunction of the Rarita-Schwinger projector. Here, the explicit expression of the Euclidean Rarita-Schwinger projector is given by

$$
\mathbf{P}^{\mu \nu}:=\Lambda^{+}\left(-\delta^{\mu \nu}+\frac{1}{3} \gamma^{\mu} \gamma^{\nu}-\frac{2}{3} \frac{P^{\mu} P^{\nu}}{M^{2}}+\frac{i}{3} \frac{P^{\mu} \gamma^{\nu}-P^{\nu} \gamma^{\mu}}{M}\right)=: \Lambda^{+} \Lambda^{\mu \nu} .
$$

The most general form which fulfills condition (21) is

$$
\Psi_{10}^{\mu \nu}(p ; P)=D_{i} \mathcal{S}_{i} \Lambda^{+} \Lambda^{\mu \nu}+i E_{i} \gamma_{5} \mathcal{A}_{i}^{\mu} \Lambda^{+} \hat{p}_{T}^{\lambda} \Lambda^{\lambda \nu}
$$

which requires the covariants again to be eigenfunctions of $\Lambda^{+}$, as a consequence we may use the same choice for the $\mathcal{A}_{i}^{\mu}$ and $\mathcal{S}_{i}$ as in the octet case and are left with eight independent scalar functions.

In the rest frame of the bound state, the decuplet wave functions are then denoted by

$$
\begin{aligned}
\Psi_{10}^{i j}(p ; P)= & \left(\begin{array}{cc}
\left(\delta^{i j}-\frac{1}{3} \sigma^{i} \sigma^{j}\right) D_{1} & 0 \\
\frac{1}{p}(\boldsymbol{\sigma} \boldsymbol{p})\left(\delta^{i j}-\frac{1}{3} \sigma^{i} \sigma^{j}\right) D_{2} & 0
\end{array}\right)+ \\
& +\left(\begin{array}{cc}
\left(-\hat{p}^{i} E_{4}+i(\boldsymbol{\sigma} \times \hat{\boldsymbol{p}})^{i} E_{6}\right)\left(\hat{p}^{j}-\frac{1}{3}(\boldsymbol{\sigma} \hat{\boldsymbol{p}}) \sigma^{j}\right) & 0 \\
\frac{1}{p}\left(-\hat{p}^{i} E_{3}+i(\boldsymbol{\sigma} \times \hat{\boldsymbol{p}})^{i} E_{5}\right)(\boldsymbol{\sigma} \boldsymbol{p})\left(\hat{p}^{j}-\frac{1}{3}(\boldsymbol{\sigma} \hat{\boldsymbol{p}}) \sigma^{j}\right) & 0
\end{array}\right) \\
\Psi_{10}^{4 j}(p ; P)= & \left(\begin{array}{cc}
\frac{i}{p}\left(p^{j}-\frac{1}{3}(\boldsymbol{\sigma} \boldsymbol{p}) \sigma^{j}\right) E_{2} & 0 \\
i(\boldsymbol{\sigma} \hat{\boldsymbol{p}})\left(\hat{p}^{j}-\frac{1}{3}(\boldsymbol{\sigma} \hat{\boldsymbol{p}}) \sigma^{j}\right) E_{1} & 0
\end{array}\right)
\end{aligned}
$$

and all other components of $\Psi_{10}^{\mu \nu}(p ; P)$ vanish. The appearance of $\gamma_{5}$ in eq. (23) has interchanged upper and lower components as compared to the octet case. 
Instead of working with the Bethe-Salpeter wave functions one may alternatively use the Bethe-Salpeter vertex functions obtained by amputating the external quark and diquark propagators from the wave function:

$$
\begin{aligned}
\Phi_{8}(p ; P) & =S^{-1}\left(p_{a}\right) D^{-1}\left(p_{b}\right) \Psi_{8}(p ; P), \\
\Phi_{8}^{\mu}(p ; P) & =S^{-1}\left(p_{a}\right)\left(D^{-1}\right)^{\mu \nu}\left(p_{b}\right) \Psi_{8}^{\nu}(p ; P), \\
\Phi_{10}^{\mu \rho}(p ; P) & =S^{-1}\left(p_{a}\right)\left(D^{-1}\right)^{\mu \nu}\left(p_{b}\right) \Psi_{10}^{\nu \rho}(p ; P),
\end{aligned}
$$

Substituting the wave functions by the vertex functions in the Bethe-Salpeter equations (7.8) leads to a reformulation of the bound state equations which is sometimes more convenient. For example in ref. [30, 1] the equations containing only scalar diquarks have been solved in a form which includes the vertex function explicitly.

\subsection{Orbital Angular Momentum and Spin of the Bethe-Salpeter Wave Functions in the Rest Frame of the Bound State}

Whereas the choice of the covariants in (15, 16) which build the octet and decuplet wave functions is well suited for numerical computation and further covariant calculations, their physical interpretation is not obvious.

In general, covariant wave functions possess only the mass of the bound state $M$ and its total angular momentum $J$ as good quantum numbers. In the rest frame of the bound state, however, the wave functions can be written as a sum of tri-spinors each possessing definite orbital angular momentum and spin, thus allowing a direct interpretation of the different components. These tri-spinors are linear combinations of the covariants $\mathcal{S}_{i}$ and $\mathcal{A}_{i}$ which have been constructed in the previous subsection, multiplied by the respective Dirac matrices $\left(\gamma_{5} C\right)_{\beta \gamma}$ and $\left(\gamma^{\mu} C\right)_{\beta \gamma}$ denoting the diquark content, respectively.

In the rest frame the Pauli-Lubanski operator for a tri-spinor is given by

$$
W^{i}=\frac{1}{2} \epsilon_{i j k} \mathcal{L}^{j k}
$$

whose square characterises the total angular momentum

$$
W^{i} W^{i} \psi_{\alpha \beta \gamma}=J(J+1) \psi_{\alpha \beta \gamma}
$$

Here, $\psi_{\alpha \beta \gamma}$ is the tri-spinor wave function with positive parity and positive energy. The tensor $\mathcal{L}^{j k}$ is the sum of an orbital part, $L^{j k}$, and a spin part, $S^{j k}$, which read

$$
\begin{aligned}
L^{j k}= & \sum_{a=1}^{3}(-i)\left(p_{a}^{j} \frac{\partial}{\partial p_{a}^{k}}-p_{a}^{k} \frac{\partial}{\partial p_{a}^{j}}\right) \\
2\left(S^{j k}\right)_{\alpha \alpha^{\prime}, \beta \beta^{\prime}, \gamma \gamma^{\prime}}= & \left(\sigma^{j k}\right)_{\alpha \alpha^{\prime}} \otimes \delta_{\beta \beta^{\prime}} \otimes \delta_{\gamma \gamma^{\prime}}+\delta_{\alpha \alpha^{\prime}} \otimes\left(\sigma^{j k}\right)_{\beta \beta^{\prime}} \otimes \delta_{\gamma \gamma^{\prime}}+ \\
& \delta_{\alpha \alpha^{\prime}} \otimes \delta_{\beta \beta^{\prime}} \otimes\left(\sigma^{j k}\right)_{\gamma \gamma^{\prime}}
\end{aligned}
$$


such that $\mathcal{L}^{j k}=L^{j k}+\frac{1}{2} S^{j k}$. Obviously, $L^{j k}$ is proportional to the unit matrix in Dirac space. The definition of $\sigma^{\mu \nu}:=-\frac{i}{2}\left[\gamma^{\mu}, \gamma^{\nu}\right]$ differs by a minus sign from its Minkowski counterpart. The tensors $L$ and $S$ are written as a sum over the respective tensors for each of the three constituent quarks which are labelled $a=1 \ldots 3$ and with respective Dirac indices $\alpha \alpha^{\prime}, \beta \beta^{\prime}, \gamma \gamma^{\prime}$.

With the definition of the spin matrix $\Sigma^{i}=\frac{1}{2} \epsilon_{i j k} \sigma^{j k}$ the Pauli-Lubanski operator reads

$$
\begin{aligned}
\left(W^{i}\right)_{\alpha \alpha^{\prime}, \beta \beta^{\prime}, \gamma \gamma^{\prime}}= & L^{i} \delta_{\alpha \alpha^{\prime}} \otimes \delta_{\beta \beta^{\prime}} \otimes \delta_{\gamma \gamma^{\prime}}+\left(S^{i}\right)_{\alpha \alpha^{\prime}, \beta \beta^{\prime}, \gamma \gamma^{\prime}} \\
L^{i}= & \left(-i \epsilon_{i j k} p^{j} \frac{\partial}{\partial p^{k}}\right. \\
\left(S^{i}\right)_{\alpha \alpha^{\prime}, \beta \beta^{\prime}, \gamma \gamma^{\prime}}= & \frac{1}{2}\left(\left(\Sigma^{i}\right)_{\alpha \alpha^{\prime}} \otimes \delta_{\beta \beta^{\prime}} \otimes \delta_{\gamma \gamma^{\prime}}+\delta_{\alpha \alpha^{\prime}} \otimes\left(\Sigma^{i}\right)_{\beta \beta^{\prime}} \otimes \delta_{\gamma \gamma^{\prime}}+\right. \\
& \left.\delta_{\alpha \alpha^{\prime}} \otimes \delta_{\beta \beta^{\prime}} \otimes\left(\Sigma^{i}\right)_{\gamma \gamma^{\prime}}\right)
\end{aligned}
$$

where we have already introduced the relative momentum $p$ between quark and diquark via a canonical transformation:

$$
P=p^{1}+p^{2}+p^{3}, \quad p=\eta\left(p^{1}+p^{2}\right)-(1-\eta) p^{3}, \quad p^{\prime}=\frac{1}{2}\left(p^{1}-p^{2}\right) .
$$

Assuming a pointlike diquark, the relative momentum between quark 1 and $2, p^{\prime}$, vanishes and the only contribution to the orbital angular momentum stems from $p$. $\mathbf{W}^{2}$ now takes the form

$$
\begin{aligned}
\mathbf{W}^{2}= & \mathbf{L}^{2}+2 \boldsymbol{L} \cdot \boldsymbol{S}+\mathbf{S}^{2}, \\
\mathbf{L}^{2}= & \left(2 p^{i} \frac{\partial}{\partial p^{i}}-\boldsymbol{p}^{2} \Delta_{p}+p^{i} p^{j} \frac{\partial}{\partial p^{i}} \frac{\partial}{\partial p^{j}}\right) \\
2(\mathbf{L} \cdot \mathbf{S})_{\alpha \alpha^{\prime}, \beta \beta^{\prime}, \gamma \gamma^{\prime}}= & -\epsilon_{i j k} p^{j} \frac{\partial}{\partial p^{k}}\left(\left(\Sigma^{i}\right)_{\alpha \alpha^{\prime}} \otimes \delta_{\beta \beta^{\prime}} \otimes \delta_{\gamma \gamma^{\prime}}+\right. \\
& \left.\delta_{\alpha \alpha^{\prime}} \otimes\left[\left(\Sigma^{i}\right)_{\beta \beta^{\prime}} \otimes \delta_{\gamma \gamma^{\prime}}+\delta_{\beta \beta^{\prime}} \otimes\left(\Sigma^{i}\right)_{\gamma \gamma^{\prime}}\right]\right), \\
\left(\mathbf{S}^{2}\right)_{\alpha \alpha^{\prime}, \beta \beta^{\prime}, \gamma \gamma^{\prime}}= & \frac{1}{4}\left(9 \delta_{\alpha \alpha^{\prime}} \otimes \delta_{\beta \beta^{\prime}} \otimes \delta_{\gamma \gamma^{\prime}}+2 \delta_{\alpha \alpha^{\prime}} \otimes\left[\left(\Sigma^{i}\right)_{\beta \beta^{\prime}} \otimes \delta_{\gamma \gamma^{\prime}}+\delta_{\beta \beta^{\prime}} \otimes\left(\Sigma^{i}\right)_{\gamma \gamma^{\prime}}\right]+\right. \\
& \left.+2 \delta_{\alpha \alpha^{\prime}} \otimes\left(\Sigma^{i}\right)_{\beta \beta^{\prime}} \otimes\left(\Sigma^{i}\right)_{\gamma \gamma^{\prime}}\right) .
\end{aligned}
$$

When applying $\mathbf{W}^{2}$ to the wave functions we first note that a scalar function does not contribute to the angular momentum, e.g.

$$
\mathbf{L}^{2} S_{1}\left(p^{2}, i M p_{4}\right)=2 \boldsymbol{L} \cdot \boldsymbol{S} S_{1}\left(p^{2}, i M p_{4}\right)=0 .
$$

In table 2 we express all terms of the octet wave function in terms of eigenstates of $\mathbf{L}^{2}$ and $\mathbf{S}^{2}$. To this end we define the following linear combinations of matrices:

$$
\mathcal{B}_{1}^{\mu}=\mathcal{A}_{5}^{\mu}+\mathcal{A}_{3}^{\mu}
$$




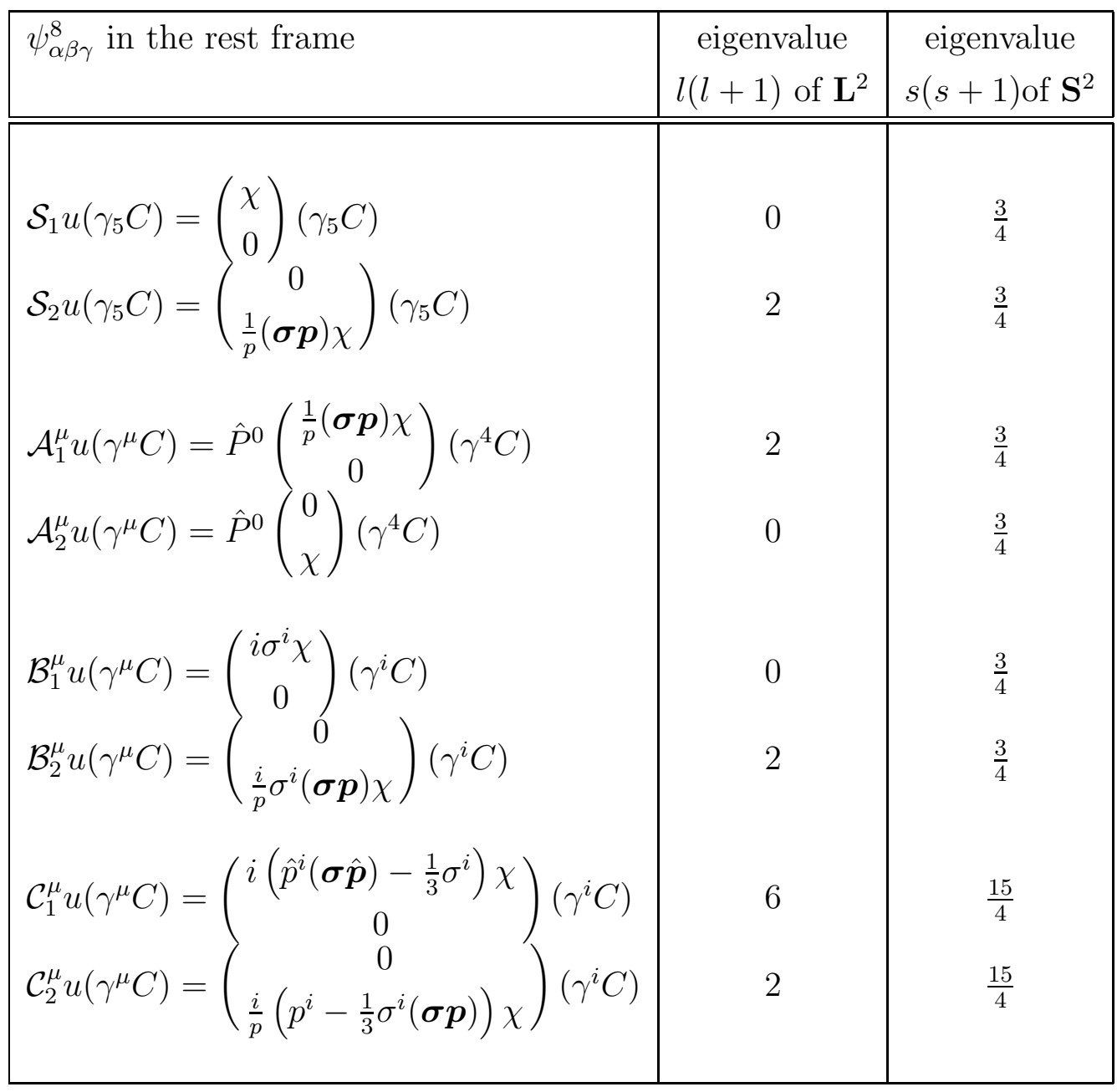

Table 2: Classification of the components of the octet wave function in terms of eigenstates of $\mathbf{L}^{2}$ and $\mathbf{S}^{2}$ in the rest frame of the bound state.

$$
\begin{aligned}
\mathcal{B}_{2}^{\mu} & =\mathcal{A}_{6}^{\mu}+\mathcal{A}_{4}^{\mu} \\
\mathcal{C}_{1}^{\mu} & =-\frac{1}{3} \mathcal{A}_{5}^{\mu}+\frac{2}{3} \mathcal{A}_{3}^{\mu}, \\
\mathcal{C}_{2}^{\mu} & =-\frac{1}{3} \mathcal{A}_{6}^{\mu}+\frac{2}{3} \mathcal{A}_{4}^{\mu} .
\end{aligned}
$$

The eigenvalue of $\mathbf{W}^{2}$ is $\frac{3}{4}$ for all terms, of course. In the table, $\chi=\left\{\chi^{+}, \chi^{-}\right\}$denotes an arbitrary Pauli two-component spinor which is the positive energy basis for quark $a$ with Dirac index $\alpha$. To derive this, the following relations between Dirac matrices have proven to be useful,

$$
\begin{gathered}
\Sigma^{j}\left(\gamma_{5} C\right)+\left(\gamma_{5} C\right)\left(\Sigma^{j}\right)^{T}=0 \\
\Sigma^{j}\left(\begin{array}{c}
\left(\gamma^{4} C\right) \\
\left(\gamma^{i} C\right)
\end{array}\right)+\left(\begin{array}{c}
\left(\gamma^{4} C\right) \\
\left(\gamma^{i} C\right)
\end{array}\right)\left(\Sigma^{j}\right)^{T}=\left(\begin{array}{c}
0 \\
2 i \epsilon_{m j i}\left(\gamma^{m} C\right)
\end{array}\right),
\end{gathered}
$$




\begin{tabular}{|c|c|c|}
\hline$\psi_{\alpha \beta \gamma}^{10}$ in the rest frame & $\begin{array}{c}\text { eigenvalue } \\
l(l+1) \text { of } \mathbf{L}^{2}\end{array}$ & $\begin{array}{c}\text { eigenvalue } \\
s(s+1) \text { of } \mathbf{S}^{2}\end{array}$ \\
\hline $\begin{array}{l}\mathcal{D}_{1} u_{\mu}\left(\gamma^{\mu} C\right)=\left(\begin{array}{c}\chi^{i} \\
0\end{array}\right)\left(\gamma^{i} C\right) \\
\mathcal{D}_{2}^{\mu \nu} u_{\nu}\left(\gamma^{\mu} C\right)=\left(\begin{array}{c}0 \\
\frac{1}{p}\left((\boldsymbol{\sigma} \boldsymbol{p}) \chi^{i}-\frac{2}{3} \sigma^{i}(\boldsymbol{p} \boldsymbol{\chi})\right)\end{array}\right)\left(\gamma^{i} C\right)\end{array}$ & 0 & $\frac{15}{4}$ \\
\hline $\begin{array}{l}\mathcal{E}_{1}^{\mu \nu} u_{\nu}\left(\gamma^{\mu} C\right)=i \hat{P}^{0}\left(\begin{array}{c}\frac{1}{p}(\boldsymbol{p} \boldsymbol{\chi}) \\
0 \\
0\end{array}\right)\left(\gamma^{4} C\right) \\
\mathcal{E}_{2}^{\mu \nu} u_{\nu}\left(\gamma^{\mu} C\right)=i \hat{P}^{0}\left(\begin{array}{c}(\boldsymbol{\sigma} \hat{\boldsymbol{p}})(\hat{\boldsymbol{p}} \boldsymbol{\chi})\end{array}\right)\left(\gamma^{4} C\right)\end{array}$ & 2 & $\frac{3}{4}$ \\
\hline $\begin{array}{l}\mathcal{E}_{3}^{\mu \nu} u_{\nu}\left(\gamma^{\mu} C\right)=\left(\begin{array}{c}\sigma^{i}(\boldsymbol{\sigma} \hat{\boldsymbol{p}})(\hat{\boldsymbol{p}} \boldsymbol{\chi}) \\
0 \\
0 \\
\mathcal{E}_{4}^{\mu \nu} u_{\nu}\left(\gamma^{\mu} C\right)=\left(\gamma^{i} C\right) \\
\frac{1}{p} \sigma^{i}(\boldsymbol{p} \boldsymbol{\chi})\end{array}\right)\left(\gamma^{i} C\right)\end{array}$ & 6 & $\frac{3}{4}$ \\
\hline $\begin{array}{l}\mathcal{E}_{5}^{\mu \nu} u_{\nu}\left(\gamma^{\mu} C\right)=\left(\begin{array}{c}\hat{p}^{i}(\hat{\boldsymbol{p}} \boldsymbol{\chi})-\frac{1}{3}\left[\chi^{i}+\sigma^{i}(\boldsymbol{\sigma} \hat{\boldsymbol{p}})(\hat{\boldsymbol{p}} \boldsymbol{\chi})\right] \\
0\end{array}\right)\left(\gamma^{i} C\right) \\
\mathcal{E}_{6}^{\mu \nu} u_{\nu}\left(\gamma^{\mu} C\right)=\left(\begin{array}{c}0 \\
\frac{1}{p}\left(p^{i}(\boldsymbol{\sigma} \hat{\boldsymbol{p}})(\hat{\boldsymbol{p}} \boldsymbol{\chi})-\frac{1}{5}\left[\sigma^{i}(\boldsymbol{p} \boldsymbol{\chi})+(\boldsymbol{\sigma} \boldsymbol{p}) \chi^{i}\right]\right)\end{array}\right)\left(\gamma^{i} C\right)\end{array}$ & 6 & $\frac{15}{4}$ \\
\hline
\end{tabular}

Table 3: Classification of the components of the decuplet wave function in terms of eigenstates of $\mathbf{L}^{2}$ and $\mathbf{S}^{2}$ in the rest frame of the bound state.

$$
\begin{gathered}
\Sigma^{j}\left(\gamma_{5} C\right)\left(\Sigma^{j}\right)^{T}=-3\left(\gamma_{5} C\right), \\
\Sigma^{j}\left(\begin{array}{c}
\left(\gamma^{4} C\right) \\
\left(\gamma^{i} C\right)
\end{array}\right)\left(\Sigma^{j}\right)^{T}=\left(\begin{array}{c}
-3\left(\gamma^{4} C\right) \\
\left(\gamma^{i} C\right)
\end{array}\right) .
\end{gathered}
$$

Three covariants can be regarded as "s-wave" components, $\mathcal{S}_{1}, \mathcal{A}_{2}$ and $\mathcal{B}_{1}$, and we expect the corresponding scalar functions to dominate the wave function decomposition. The other " $p$-, $d$-wave" components represent all remaining possibilities of combining orbital angular momentum between quark and diquark and the joint spin of axialvector diquark and quark to total spin $1 / 2$. In this sense the description is closed.

The individual terms of the decuplet wave function can be classified accordingly with the help of the linear combinations:

$$
\begin{aligned}
\mathcal{D}_{1} & =\mathcal{S}_{1} \\
\mathcal{D}_{2}^{\mu \nu} & =\mathcal{S}_{2} \delta^{\mu \nu}+\frac{2 i}{3} \gamma_{5}\left(\mathcal{A}_{5}^{\mu}+\mathcal{A}_{3}^{\mu}\right) \hat{p}_{T}^{\nu}
\end{aligned}
$$




$$
\begin{aligned}
\mathcal{E}_{1}^{\mu \nu} & =i \gamma_{5} \mathcal{A}_{2}^{\mu} \hat{p}_{T}^{\nu}, \\
\mathcal{E}_{2}^{\mu \nu} & =i \gamma_{5} \mathcal{A}_{1}^{\mu} \hat{p}_{T}^{\nu}, \\
\mathcal{E}_{3}^{\mu \nu} & =-i \gamma_{5}\left(\mathcal{A}_{6}^{\mu}+\mathcal{A}_{4}^{\mu}\right) \hat{p}_{T}^{\nu} \\
\mathcal{E}_{4}^{\mu \nu} & =-i \gamma_{5}\left(\mathcal{A}_{5}^{\mu}+\mathcal{A}_{3}^{\mu}\right) \hat{p}_{T}^{\nu} \\
\mathcal{E}_{5}^{\mu \nu} & =\left(-\frac{2 i}{3} \gamma_{5} \mathcal{A}_{4}^{\mu}+\frac{i}{3} \gamma_{5} \mathcal{A}_{6}^{\mu}\right) \hat{p}_{T}^{\nu}-\frac{1}{3} \mathcal{S}_{1} \delta^{\mu \nu}, \\
\mathcal{E}_{6}^{\mu \nu} & =\left(-\frac{4 i}{5} \gamma_{5} \mathcal{A}_{3}^{\mu}+\frac{i}{5} \gamma_{5} \mathcal{A}_{5}^{\mu}\right) \hat{p}_{T}^{\nu}-\frac{1}{5} \mathcal{S}_{2} \delta^{\mu \nu} .
\end{aligned}
$$

The result can be found in table 3. Due to the spin projection by use of the RaritaSchwinger projector the eigenvalue of $\mathbf{W}^{2}$ is $\frac{15}{4} . \quad \chi^{i}=\left\{\chi^{+i}, \chi^{-i}\right\}(i=1,2,3)$ denotes a two-component vector-spinor which survives the spin-3/2 projection in the rest frame. The Rarita-Schwinger constraints reduce to $\boldsymbol{\sigma} \boldsymbol{\chi}=0$.

For the decuplet only one $s$-wave component exists, $\mathcal{D}_{1}$. Again these covariants exhaust all possible couplings of spin and orbital angular momentum, note that even an orbital angular momentum $l=3$ contributes to a spin-3/2 state, $\mathcal{E}_{6}$. A contribution of an $l=1$

state with scalar diquark, such as $\left(\begin{array}{c}0 \\ (\boldsymbol{p} \boldsymbol{\chi})\end{array}\right)\left({ }_{5} C\right)$, is forbidden by the Pauli principle for pure decuplet states, but may admix in the case of broken flavour symmetry.

\section{Ladder approximation to the Bethe-Salpeter equa- tion}

In our preceding publication [1] we used a momentum routing in the Bethe-Salpeter equation, where the interaction (propagator of the exchanged quark) is manifestly independent of the total momentum $P$ of the baryon, i.e. $q=-p-p^{\prime}$. A $P$-independent kernel is desirable for the following reasons: As will be discussed in the next section, it reduces the numerical work when solving the integral equation quite drastically. Furthermore, the canonical normalisation condition for the Bethe-Salpeter wave function 442 becomes much more involved when the interaction is $P$-dependent. Therefore, when solving a Bethe-Salpeter equation in ladder approximation one usually tries to find a momentum routing having this very convenient feature. In our equations, however, where quark and diquark interact through quark exchange, which changes the identity of the particles after each interaction (a diquark becomes a quark and a quark becomes a diquark after the quark exchange), demanding a $P$-independent interaction defines a "modified ladder approximation" with results deviating from other momentum routings. Note, that this problem does not occur in the Bethe-Salpeter approach if the interaction, e.g. meson exchange in the two nucleon system or gluon exchange between quark and antiquark, does 

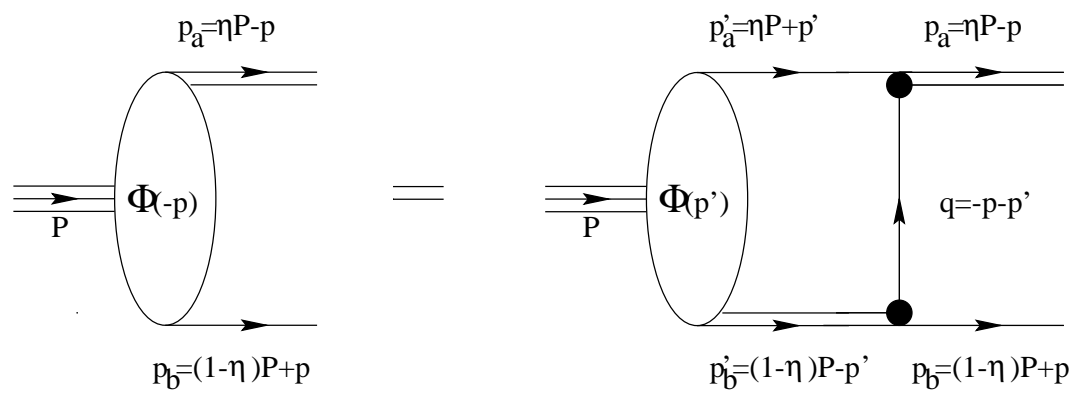

Figure 1: Momentum routing defining the modified ladder approximation.

not change the identity of the constituents. Almost all Bethe-Salpeter equations treated in the literature are of this kind. For a comprehensive review of the existing Bethe-Salpeter literature see 443. In order to clarify the ambiguity arising in the diquark-quark BetheSalpeter equation, which came up during our investigations, we discuss here two possible momentum routings and in section 6 and appendix $B$ the corresponding variations of the results.

For sake of clarity of this discussion we temporarily restrict ourselves to the part of the octet equation which involves only scalar diquarks, i.e. eq. (77) with $g_{a}=0$. Furthermore, it is more transparent to work with the Bethe-Salpeter equation which involves the vertex function (see eq. (25)):

$$
\Phi_{8}(p ; P)=-\left|g_{s}\right|^{2} \int \frac{d^{4} p^{\prime}}{(2 \pi)^{4}} \gamma_{5} \tilde{S}(-q) \gamma_{5} S\left(p_{a}^{\prime}\right) D\left(p_{b}^{\prime}\right) \Phi_{8}\left(p^{\prime} ; P\right) .
$$

Repeating the procedure discussed in section 3.1 for the vertex instead of the wave function leads to an ansatz [30, 1]

$$
\Phi_{8}(p ; P)=\left(\begin{array}{cc}
\mathbf{1} \hat{S}_{1}(p, z) & 0 \\
\frac{1}{p}(\boldsymbol{\sigma} \boldsymbol{p}) \hat{S}_{2}(p, z) & 0
\end{array}\right) .
$$

Note that in the lower component of this spinor-like object the spin is orientated along the spatial part of the relative momentum. In the Bethe-Salpeter equation (47) the momentum of the constituent quark is given by $p_{a}^{\prime}=p^{\prime}+\eta P$, and the momentum of the scalar diquark by $p_{b}^{\prime}=-p^{\prime}+(1-\eta) P$. $P$ denotes the total momentum of the nucleon and $p$ the relative momentum between quark and diquark. The Mandelstam parameter $\eta$ describes how $P$ is partitioned to quark and diquark. The fact that the eigenvalues of the Bethe-Salpeter equation are independent of $\eta$ is a direct consequence of Lorentz covariance.

If the momentum of the exchanged quark is chosen as $q=-p-p^{\prime}$, the interaction kernel is by construction independent of the total momentum. Since the quark exchange transforms a quark to a diquark and vice versa, after the interaction, the relative momentum between these particles becomes $(1-\eta) p_{a}-\eta p_{b}=-p$, see fig. 1 for this momentum 
routing. We now demand $\Phi_{8}(-p ; P)=\Phi_{8}(\boldsymbol{p},-z, P)$, i.e. the orientation of the nucleon spin should not depend on the orientation of $p$ as required by the Dirac decomposition before projection onto positive energies. Then the vertex function appearing on the left hand side in eq. (47) reads

$$
\Phi_{8}(p ; P)=\left(\begin{array}{cc}
\mathbf{1} \hat{S}_{1}(p,-z) & 0 \\
\frac{1}{p}(\boldsymbol{\sigma} \boldsymbol{p}) \hat{S}_{2}(p,-z) & 0
\end{array}\right) .
$$

The definition of the momentum $q$ together with eq. (49) is what we call "modified ladder approximation" which leads to satisfying results for various nucleon form factors in the weak binding regime, as can be seen in ref. [1]. Using such a prescription we have been able to reproduce the results of ref. [30].

Another possible momentum routing allows $P$ to flow through the quark exchange, $q=-p-p^{\prime}+(1-2 \eta) P$, see fig. 2. When taking not into account, that quark and diquark change their role after exchanging a quark, that means attributing the index "a" to the quark and index "b" to the diquark, the relative momentum after the interaction is given by $p$. Correspondingly, the vertex function on the left hand side in eq. (47) is denoted by

$$
\Phi_{8}(p ; P)=\left(\begin{array}{cc}
\mathbf{1} \hat{S}_{1}(p, z) & 0 \\
\frac{1}{p}\left(\boldsymbol{\sigma} \boldsymbol{p}^{\prime}\right) \hat{S}_{2}(p, z) & 0
\end{array}\right) .
$$

We name this "direct ladder approach". Although one might expect that both choices of the momentum routing lead to the same physical results, we found that this is actually not the case. In the next sections and appendix $\mathbb{B}$ we report on calculations using both momentum routings, see also [44] for further results obtained in the direct ladder approach. We found that physical results slightly differ for these two choices. We will show, however, that both possibilities are manifestly Lorentz covariant, i.e. the eigenvalues do not depend on the Mandelstam parameter $\eta$. Furthermore, for weak binding the eigenvalues almost coincide. Given that the ladder approximation is only reliable for weak binding we conclude that both methods are of similar validity.
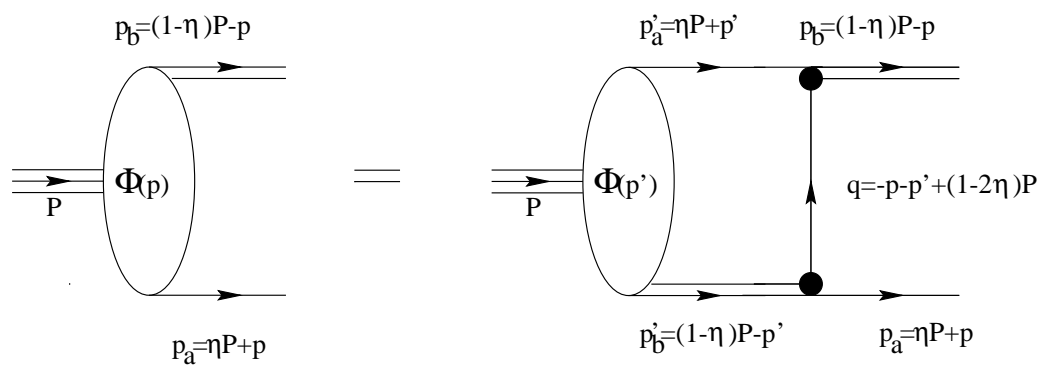

Figure 2: Momentum routing defining the direct ladder approximation. 
Although during this discussion we restricted ourselves for clarity to scalar diquarks and to the Bethe-Salpeter equation involving the vertex function, it can be extended in a straightforward way to the complete equation including the axialvector diquark channel, the decuplet equation and also to the equations $(7,8)$ involving the wave functions in an obvious way. The reported numerical results are always given for the full problem.

\section{$5 \quad$ Numerical method}

For the numerical solutions of the Bethe-Salpeter equations we developed an iterative hybrid algorithm, which allows a very efficient and fast computation. A description of our numerical method can be found in a forthcoming publication which presents this algorithm in all details [40. Thus we will focus here on the main steps only.

We solve the Bethe-Salpeter equation as a system of equations for the wave function $\Psi$ and the vertex function $\Phi$, see eq. (77, 8). Both wave and vertex function can be expanded in the rest frame according to eq. (17, 24). Although there are 10 equations for the eight octet functions, stemming from 2 equations in the scalar diquark channel and $2 \times 4$ equations in the axialvector diquark channel, we confirmed that two of them are redundant. The decuplet system yields $2 \times 12$ equations for eight scalar functions due to the tensor character of the wave function which reduce again to eight independent equations. This especially underlines the necessity of including the subdominant amplitudes describing orbital angular momentum to keep the system closed.

We expand the scalar functions (amplitudes) $S, A$ and $D, E$ defined in (14, 23) into Chebyshev polynomials of the second kind,

$$
\begin{aligned}
& Y_{i}(P ; p)=\sum_{n=0}^{\infty} i^{n} Y_{i}^{n}\left(P^{2} ; p^{2}\right) U_{n}(\hat{P} \cdot \hat{p}) \\
& \hat{Y}_{i}(P ; p)=\sum_{n=0}^{\infty} i^{n} \hat{Y}_{i}^{n}\left(P^{2} ; p^{2}\right) U_{n}(\hat{P} \cdot \hat{p}),
\end{aligned}
$$

where amplitudes with a hat, i.e. $\hat{Y}_{i}$, belong to the vertex function and the ones without a hat to the wave function. Here we use the generic label $Y_{i}$ according to $Y_{i}=$ $\left\{S_{1}, S_{2}, \ldots, A_{6}\right\}_{\text {octet }},\left\{D_{1}, D_{2}, \ldots, E_{6}\right\}_{\text {decuplet }}$.

Throughout the calculation we work with usual hyperspherical coordinates,

$$
\left(\begin{array}{c}
p_{1}^{\prime} \\
p_{2}^{\prime} \\
p_{3}^{\prime} \\
p_{4}^{\prime}
\end{array}\right)=p^{\prime}\left(\begin{array}{c}
\sin \psi^{\prime} \sin \theta^{\prime} \sin \phi^{\prime} \\
\sin \psi^{\prime} \sin \theta^{\prime} \cos \phi^{\prime} \\
\sin \psi^{\prime} \cos \theta^{\prime} \\
\cos \psi^{\prime}
\end{array}\right), \quad z^{\prime}=\cos \psi^{\prime}=\hat{P} \cdot \hat{p}^{\prime}
$$


We are free to choose the spatial part of the relative momentum $p$ appearing on the l.h.s. of the Bethe-Salpeter equation (7). Without loss of generality, we select $p^{\mu}=$ $\left(0,0, p \sqrt{1-z^{2}}, p z\right)$.

We expand quark and diquark propagators into Chebyshev polynomials as well and project the Bethe-Salpeter equation onto the Chebyshev moments of the amplitudes, $\hat{Y}_{i}^{m}, Y_{i}^{m}$. Note that in the chosen Lorentz frame with the relative momentum $\mathbf{p}$ parallel to the third axis this is especially easy because in this case the amplitudes as given in (17) do not mix. The integration necessary to generate the kernel will be performed in hyperspherical coordinates. The integrations over $\phi^{\prime}$ and $\theta^{\prime}$ are done analytically, and the remaining two over $z^{\prime}$ and $z$ (due to the projection) numerically.

The final equation suitable for iteration or diagonalisation reads:

$$
\begin{aligned}
Y_{i}^{m}\left(p_{l_{1}}\right) & =-g^{2} \sum_{j=1}^{8} \sum_{n=0}^{n_{\max }} P_{i j}^{m n}\left(p_{l_{1}}\right) \hat{Y}_{j}^{n}\left(p_{l_{1}}\right) \\
\hat{Y}_{j}^{n}\left(p_{l_{1}}\right) & =\sum_{k=1}^{8} \sum_{m=0}^{m_{\max }} \int_{0}^{\infty} d p^{\prime} p^{\prime 3} H_{j k}^{n m}\left(p_{l_{1}}, p_{l_{2}}^{\prime}\right) Y_{k}^{m}\left(p_{l_{2}}^{\prime}\right) .
\end{aligned}
$$

Here, $P_{i j}^{m n}$ is the propagator matrix with amplitude indices $i, j$ and Chebyshev moment indices $m, n$ and $H_{j k}^{n m}\left(p, p^{\prime}\right)$ is the matrix of the quark exchange kernel given on a momentum grid $\left(p_{l_{1}}, p_{l_{2}}^{\prime}\right)$ with respective amplitude indices $j, k$ and Chebyshev moment indices $n, m$. The sum over amplitude indices runs from $1 \ldots 8$ for both octet and decuplet. $m_{\max }$ and $n_{\max }$ denote the highest Chebyshev polynomial considered in the expansion of the vertex and the wave function amplitudes, respectively. The kernel includes also the flavour factors and the ratio $g_{a} / g_{s}$ (for spin- $1 / 2$ baryons). It is only in the modified ladder approximation that the integral kernel does not include an explicit dependence on the bound state mass $M$ for all values of $\eta$, which makes a fast determination of $M$ for given couplings feasible. We refer to $g$ as the eigenvalue of the Bethe-Salpeter equation which is $g_{s}$ for spin- $1 / 2$ and $g_{a}$ for spin-3/2 baryons.

In the following we will investigate the convergence properties of the expansion in Chebyshev polynomials. Furthermore, the independence of the eigenvalue from the Mandelstam parameter $\eta$ will be discussed. The chosen example is the octet equation (7) with the axialvector diquark propagator diagonal in Lorentz indices and a diquark size factor of the monopole type.

For all calculations reported, a momentum mesh size of $20 \times 20$ and the inclusion of only zeroth, first and second Chebyshev moment for both vertex and wave function amplitudes is sufficient for determining the eigenvalue up to $10^{-4}$ precision (see table (4). This is also reflected in the magnitudes of the vertex amplitudes $\hat{Y}_{i}$ : Going up one Chebyshev moment suppresses the amplitudes by almost one order of magnitude. The 


\begin{tabular}{|c|c|c|c|c|}
\hline \multicolumn{5}{|c|}{ Confining Propagators, $d=10$} \\
\hline$m_{\max } n_{\max }$ & 0 & 1 & 2 & 3 \\
\hline 0 & 9.4362 & 9.1533 & 9.1417 & 9.1420 \\
\hline 1 & 9.7480 & 9.2277 & 9.2260 & 9.2265 \\
\hline 2 & 9.7568 & 9.2276 & 9.1992 & 9.1988 \\
\hline 3 & 9.7568 & 9.2277 & 9.1994 & 9.1990 \\
\hline \multicolumn{5}{|c|}{ Confining Propagators, $d=1$} \\
\hline$n_{\max }$ & 0 & 1 & 2 & 3 \\
\hline 0 & 11.3797 & 10.9507 & 10.9544 & 10.9547 \\
\hline 1 & 11.8555 & 11.1401 & 11.1704 & 11.1711 \\
\hline 2 & 11.8656 & 11.1503 & 11.1443 & 11.1445 \\
\hline 3 & 11.8656 & 11.1504 & 11.1444 & 11.1446 \\
\hline
\end{tabular}

Table 4: Octet Bethe-Salpeter Equation - eigenvalues $g_{s}$ for various $m_{\max }$ and $n_{\max }$, the maximum Chebyshev momenta of vertex and wave function. Parameters: $m_{q}=m_{0^{+}}=m_{1^{+}}$, $M=1.5 m_{q}, \Lambda=2 m_{q}, g_{a} / g_{s}=0.5, \eta=0.5$, momentum grid size $n_{p}=20$.

wave function amplitudes $Y_{i}$ converge somewhat slower, see also sect. 6.3. For a large pole screening factor, $d>5$, and a weak binding situation the even Chebyshev momenta are more pronounced than the odd ones.

Increasing the coupling constant $g_{a}$ in the nucleon equation always lowers the eigenvalue, hence the axialvector diquark enhances binding.

For tree level propagators the choice of the Mandelstam parameter $\eta$ is limited to the values $\eta \in\left[1-\min \left\{m_{0^{+}}, m_{1^{+}}\right\} / M, m_{q} / M\right]$ to avoid singularities in the propagators. This restriction should not apply for confining propagators. To demonstrate this, we choose the extreme case $m_{0^{+}}=m_{1^{+}}=2 m_{q}$ and the tree level threshold $M=3 m_{q}$. Then, tree level propagators limit the choice to $\eta=1 / 3$, as opposed to confining propagators. For a small pole screening factor $d=1$ we could vary $\eta$ in a wide range without affecting the eigenvalue, for $d=10$ due to numerical instabilities the invariance region depends on the constituent masses, displayed in figure 3 in the left panel. It can be seen that the poles in the tree level propagators are effectively screened although the invariance region for $d=10$ is restricted to $0.2 \ldots 0.42$.

Whereas the latter results were obtained in the modified ladder approximation, the

\footnotetext{
${ }^{4}$ For similar choices of quark and diquark masses we calculate the octet and decuplet masses, see sect. 6. The masses of the decuplet baryons are close to the tree level threshold.
} 

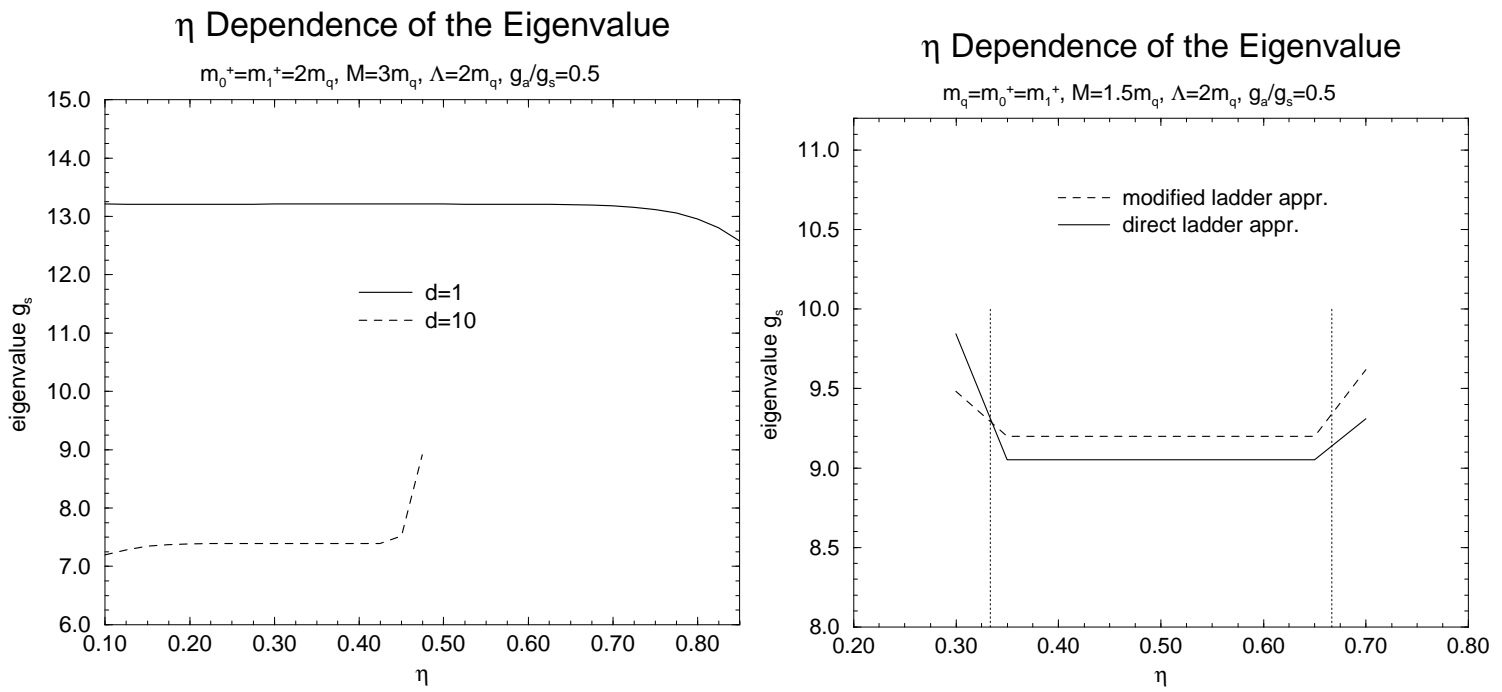

Figure 3: Eigenvalue vs. Mandelstam parameter $\eta$, left: two pole screening factors compared, right: modified and direct ladder approach compared. Dotted lines denote the location of the propagator poles.

check of $\eta$-independence for the direct ladder approach requires the total momentum $P$ to appear in the kernel: $q=-p-p^{\prime}+(1-2 \eta) P$, which slows down the numerics considerably. In the case of tree level propagators, the right panel of figure 3 shows the invariance of the eigenvalue for both momentum routings.

The crucial advantage of using confining propagators can be seen even more clearly in figure 4 . Here the function $g_{s}(M)$ decreases rapidly for tree level propagators near threshold while the corresponding function for confining propagators runs smoothly over the "pseudo"-threshold. We furthermore observe that the even Chebyshev momenta of the Bethe-Salpeter wave functions for tree level propagators close to threshold become squeezed in the low momentum domain while the corresponding odd momenta are suppressed. No such effect is present for the Bethe-Salpeter wave functions obtained with confining propagators.

All the numerical features described also apply to the decuplet equation in the weak binding regime $\left(M>\frac{3}{4}\left(m_{q}+m_{0^{+}, 1^{+}}\right)\right)$. For lower bound state masses the convergence becomes worse and for $M<\frac{1}{2}\left(m_{q}+m_{0^{+}, 1^{+}}\right)$our method failed in finding a real eigenvalue for the coupling $g_{a}$. In this mass region an effect well known from the Cutkosky model is visible: two states described by their functions $g_{a}(M)$ collide and form a complex conjugate pair of eigenvalues [45, 46]. This shortcoming of the ladder approximation provides us with an upper limit of the coupling constant $g_{a}$ for which the approximation is valid. However, the $g_{a}$ needed to describe the baryon decuplet is well below this critical value. 
Eigenvalue vs. Bound State Mass

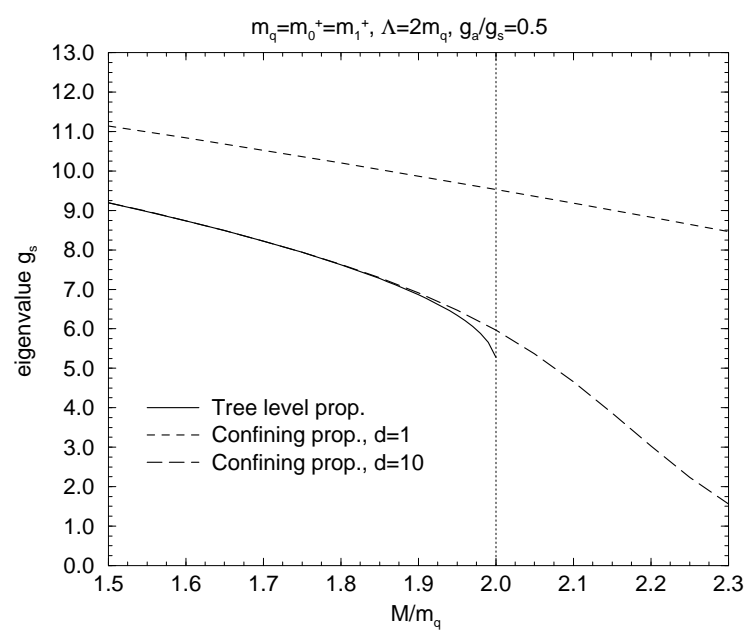

Figure 4: Eigenvalues vs. bound state mass $M$. Absence of threshold effects for confining propagators.

\section{Results: Masses and Selected Wave Functions}

\subsection{Octet and Decuplet Masses}

In our approach the strange quark constituent mass $m_{s}$ is the only source of flavour symmetry breaking. Isospin is assumed to be conserved. The equations describing octet and decuplet baryons have been derived under the premises of flavour and spin conservation, i.e. only wave function components with same spin and flavour content couple. The flavour structure of the eight equations describing $N, \Lambda, \Sigma, \Xi, \Delta, \Sigma^{*}, \Xi^{*}$ and $\Omega$ can be found in appendix $\mathrm{A}$.

In order to limit the number of parameters we assume the scalar and axialvector diquark masses to be equal. Furthermore, we choose them to be $m_{0^{+}, 1^{+}}^{f g}=\xi\left(m_{f}+m_{g}\right)$ where $f g \in\{u u, u s, s s\}$ is the flavour content of the diquark. We denote $\xi$ as diquark mass parameter. Assuming $\xi \in[0,1]$ is obviously natural.

With these choices the model has the following parameters: two constituent quark masses $m_{u}=m_{d}, m_{s}$, the pole screening factor $d$, the diquark size factor $\Lambda$, the diquark mass parameter $\xi$ and the couplings $g_{a}, g_{s}$.

We do not try to make a thorough fit onto the baryon masses in our parameter space. Inspired by our results for the form factors of proton and neutron [1], we assume first $d=10$. This results in only slight modifications of the propagators compared to the tree level ones for spacelike momenta. Furthermore, we choose $\Lambda=1 \mathrm{GeV} \approx 2 m_{u}$ and $\xi=1$ to stay close to calculations of octet and decuplet masses in NJL-diquark-models as done in [25] and [23]. These authors, however, used just a static approximation to the Bethe- 


\begin{tabular}{|l|l|l|l|l|}
\hline & exp. & \multicolumn{2}{|c|}{ mod. ladder } & dir. ladder \\
& & I & II & III \\
\hline$d$ & & 10 & 1 & 1 \\
$\Lambda \quad(\mathrm{GeV})$ & & 1 & 1 & 1 \\
$m_{u}(\mathrm{GeV})$ & & 0.5 & 0.5 & 0.5 \\
$m_{s}(\mathrm{GeV})$ & & 0.65 & 0.63 & 0.63 \\
$\xi$ & & 1 & 0.73 & 0.73 \\
\hline$g_{a}$ & & 10.35 & 10.92 & 10.05 \\
$g_{s}$ & & 9.43 & 8.06 & 7.34 \\
\hline$M_{\Lambda}(\mathrm{GeV})$ & 1.116 & 1.123 & 1.130 & 1.133 \\
$M_{\Sigma}(\mathrm{GeV})$ & 1.193 & 1.134 & 1.137 & 1.140 \\
$M_{\Xi}(\mathrm{GeV})$ & 1.315 & 1.307 & 1.319 & 1.319 \\
\hline$M_{\Sigma^{*}}(\mathrm{GeV})$ & 1.384 & 1.373 & 1.372 & 1.380 \\
$M_{\Xi^{*}}(\mathrm{GeV})$ & 1.530 & 1.545 & 1.548 & 1.516 \\
$M_{\Omega}(\mathrm{GeV})$ & 1.672 & 1.692 & 1.697 & 1.665 \\
\hline \hline$\chi^{2}$ & & 0.0028 & 0.0028 & 0.0021 \\
\hline
\end{tabular}

Table 5: Octet and decuplet masses obtained with the maximum order in Chebyshev polynomials $m_{\max }=n_{\max }=3$ and momentum grid size $n_{p}=20$.

Salpeter equation. Note that this part of our calculation has been done only in modified ladder approximation since we were forced to choose $\eta<0.4$ (cf. figure 3), and solely for $\eta=0.5$ the integral kernel does not depend on the baryon mass in the direct approach, which allows a fast determination of $M$. We then fix the couplings $g_{a}$ and $g_{s}$ by the nucleon and delta mass. The ratio $g_{a} / g_{s}$ is quite independent from the ratio $M_{\Delta} / m_{q}$ and varies weakly with $d$. Finally we vary just the two constituent quark masses to obtain the other six hyperon masses reasonably close to their experimental values.

Secondly, we explore the case $d=1$ and now additionally allow the diquark mass factor $\xi$ to vary. In [1] we showed that this strong screening of the propagator poles leads to overestimated nucleon e.m. form factors for high $Q^{2}$. In this case we also compare results for the two momentum routings, modified and direct ladder approach. In both calculations $\eta$ is set to 0.5 .

The results are given in table 5. The quality of the results may be read from the quantity $\chi^{2}=\sum_{i=1}^{6} \frac{\left(M_{t h e o r}^{i}-M_{e x p}^{i}\right)^{2}}{\left(M_{e x p}^{i}\right)^{2}}$. Note that the mean deviation of the calculated masses from the experimental ones is of the order of half a per cent or less. Column I shows the results for $d=10$. A remarkable feature is the large constituent quark mass $m_{u}=500$ $\mathrm{MeV}$, whereas the constituent mass difference between strange and up quark is $150 \mathrm{MeV}$, 
a commonly used value. In the following two subsections we will show for this set of parameters the wave function amplitudes for the nucleon and the delta and discuss the contribution of the various components with respect to their orbital angular momentum and exemplify flavour symmetry breaking effects on the wave and vertex function of the $\Lambda$-hyperon.

The next columns, columns II and III, show the results for $d=1$ with the other parameters chosen to give baryon masses to the same level of accuracy as before. In this case quark and diquark propagators are strongly modified for spacelike momenta. Quark and diquark "masses" begin to loose their meaning which we usually attach to them. This is reflected in our solutions in a rather small diquark mass parameter which renders constituent quarks and diquarks roughly equal in their masses. Direct and modified ladder approach give approximately the same results in the latter case.

In all cases the Gell-Mann-Okubo mass formula for the octet is fulfilled with an inaccuracy of less than $0.5 \%$. It should be emphasized that the mass splitting between octet and decuplet is exclusively provided by the quark exchange with the coupling strengths $g_{s}$ and $g_{a}$ and is not due to a heavier axialvector diquark as assumed in other diquark-quark models, e.g. in [21].

\subsection{Vertex Functions for the $\Lambda$-hyperon}

The $\Lambda$-hyperon appears to be of special interest. First, its measured polarisation asymmetry in the process $p \gamma \rightarrow K^{+} \Lambda$ could provide a stringent test for the diquark-quark model. As discussed in [47], there are only scalar diquarks involved in this process and in the following we will concentrate on the scalar diquark part of the vertex function.

Secondly, broken $S U(3)$-flavour symmetry induces a component of the total antisymmetric flavour singlet $\frac{1}{\sqrt{3}}[(s u) d+(u d) s+(d s) u]$ into wave and vertex function (see appendix A] As the flavour singlet is only composed of scalar diquarks and quarks, this generates two additional scalar amplitudes $\hat{S}_{1, \text { singlet }}$ and $\hat{S}_{2, \text { singlet }}$ besides the usual two from the octet $\Lambda$ state $\frac{1}{\sqrt{6}}[(s u) d-\sqrt{2}(u d) s+(d s) u]$. The vector part of the vertex function remains unchanged in flavour space, $\frac{1}{\sqrt{2}}\left([s u]^{\mu} d-[d s]^{\mu} u\right)$.

These scalar amplitudes for the vertex function are depicted in figure 5 for the pa-

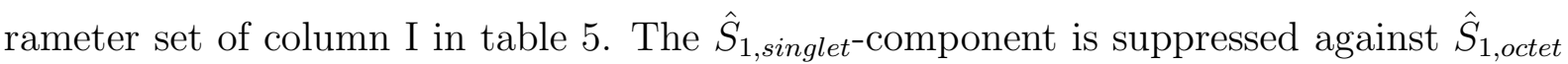
by two orders of magnitude. However, the purely relativistic $\hat{S}_{2, \text { singlet- }}$ component is only 5 times smaller than its octet counterpart. Observing that the $\hat{S}_{1}$-component usually

\footnotetext{
${ }^{5}$ With round brackets we denote scalar diquarks, e.g. $(u s)=u s-s u$. Axialvector diquarks we denote as $[u s]=u s+s u$.

${ }^{6}$ In nonrelativistic quark models with $S U(6)$ symmetry such a component is forbidden by the Pauli principle.
} 
$\Lambda$ Vertex Function: $\hat{S}_{1}(p)$

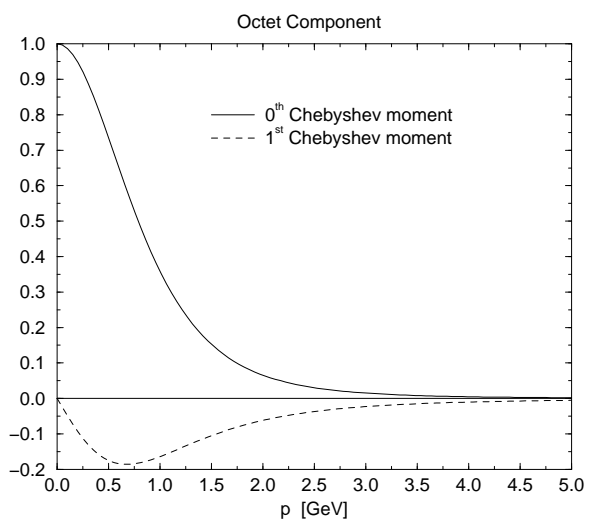

$\Lambda$ Vertex Function: $\hat{S}_{2}(p)$

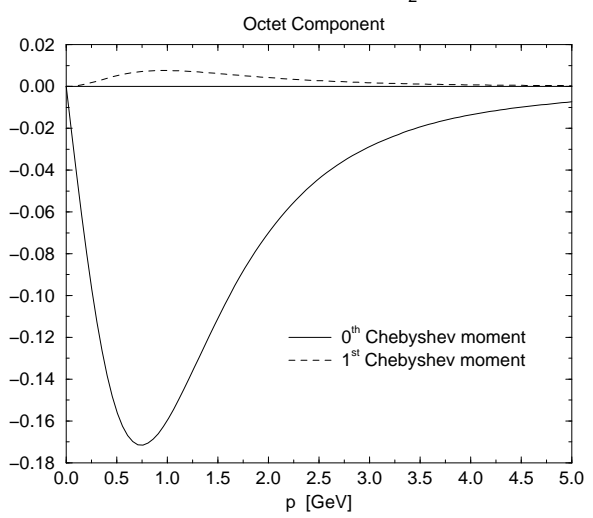

$\Lambda$ Vertex Function: $\hat{S}_{1}(p)$

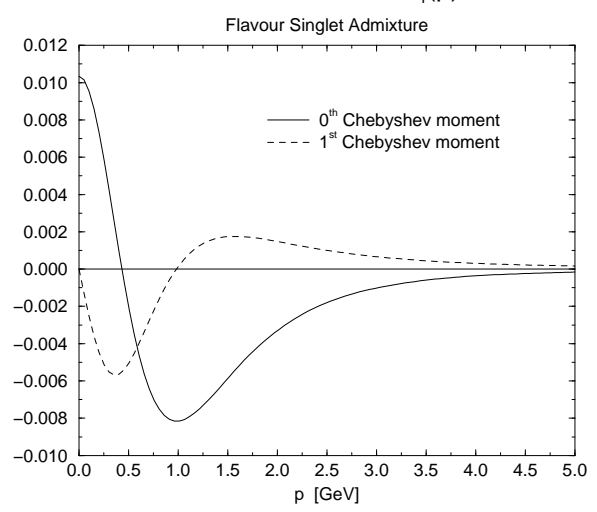

$\Lambda$ Vertex Function: $\hat{\mathrm{S}}_{2}(\mathrm{p})$

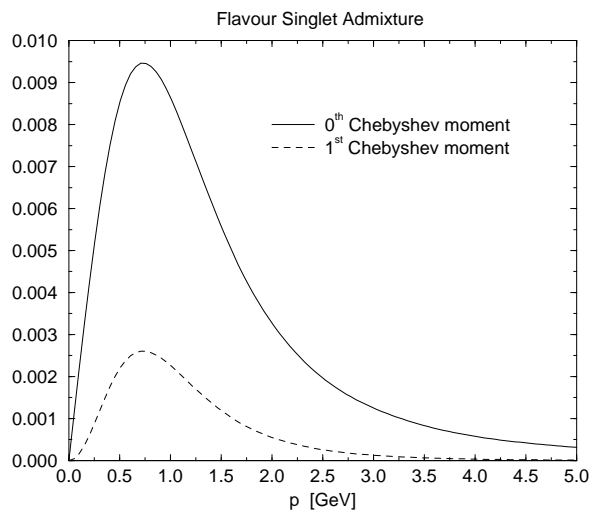

Figure 5: Scalar amplitudes of the vertex function for the $\Lambda$-hyperon, normalised to $\hat{S}_{1, \text { octet }}^{0}\left(p_{1}\right)=1$ with $p_{1}$ being the first point on the momentum mesh. The used parameters are $m_{u}=0.5 \mathrm{GeV}, m_{s}=0.65 \mathrm{GeV}, \xi=1, \eta=0.33, d=10$ and $\Lambda=1 \mathrm{GeV}$.

contributes the major part to observables as demonstrated in [1], we can safely regard the $\Lambda$-hyperon as an almost pure octet state in flavour space. 


\subsection{Wave Functions for Nucleon and Delta}

In this subsection we present Bethe-Salpeter wave functions for nucleon and delta, using the parameter set of column I in table 5. They are normalised to $S_{1}^{0}\left(p_{1}\right)=1$ (nucleon) or $D_{1}^{0}\left(p_{1}\right)=1$ (delta) where $p_{1}$ is the smallest point of the momentum mesh. The amplitudes represent the strengths of the $\left(\mathbf{L}^{2}, \mathbf{S}^{2}\right)$ eigenfunctions given in sec. 3.2 and are simple linear combinations out of the amplitudes defined in (14,23).

As already mentioned, the convergence of the wave function amplitudes in terms of Chebyshev polynomials is somewhat slower than for the vertex amplitudes: Second and zeroth Chebyshev moment of the amplitudes differ by less than one order of magnitude. All wave function amplitudes are concentrated to four-momenta $p \leq 0.6 \mathrm{GeV}$.

In figure 6, the nucleon amplitudes with even orbital angular momentum $l$ appear in the left row: These are the three $s$-waves describing (i) scalar diquark and quark, (ii,iii) axialvector diquark and quark oppositely aligned to give spin $1 / 2$. There are two axialvector diquark components due to the virtual time component of the latter. The scalar diquark component is the most important but the other $s$-waves enhance binding by approximately $30 \%$. The fourth "non-relativistic" component is a strongly suppressed $d$-wave with quark and axialvector diquark aligned to give spin $3 / 2$. The lower components depicted on the right side can be understood as the admixture of negative-energy spinors to the proton wave function and contribute approximately $10 \%$ to the binding energy.

The delta amplitudes in fig. 7 have also been arranged into "non-relativistic" (left row) and "relativistic" components (right row). As expected, the only $s$-wave dominates the decomposition, but the relativistic $p$-wave components, which act repulsively, increase the eigenvalue by approximately $20 \%$ and are thus non-negligible. Again the component with the highest orbital angular momentum, $l=3$, is highly suppressed.

No clear effects of flavour symmetry breaking, as in terms of the width in momentum space, can be detected for the other octet wave functions when compared to the nucleon. For the vertex functions (where the zeroth Chebyshev moment dominates), we observed a limited increase of the width in momentum space with increasing number of $s$-quarks (10\% difference between $N$ and $\Xi$ ). The width is hereby defined as the absolute value of the relative momentum $p$ where the zeroth Chebyshev moment of an $s$-wave amplitude reaches half the maximum value. 
Nucleon: $\mid=0, s=1 / 2-$ Scalar Diquark

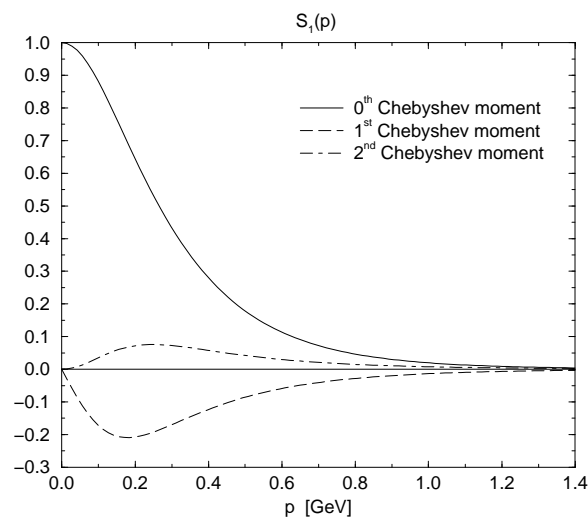

Nucleon: $I=0, s=1 / 2-A V$ diquark

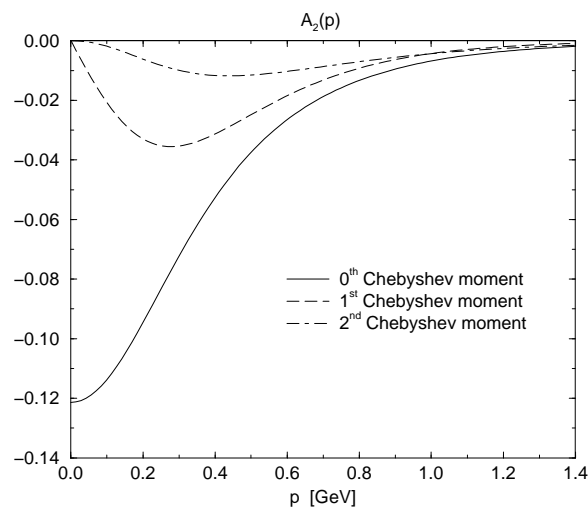

Nucleon: $l=0, s=1 / 2-A V$ Diquark

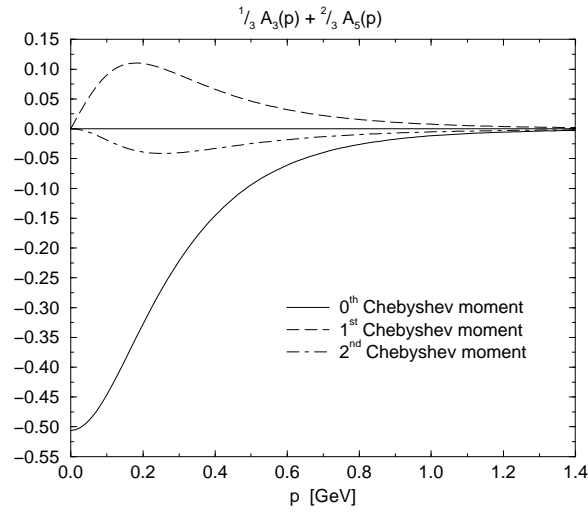

Nucleon: $I=2, s=3 / 2-A V$ Diquark

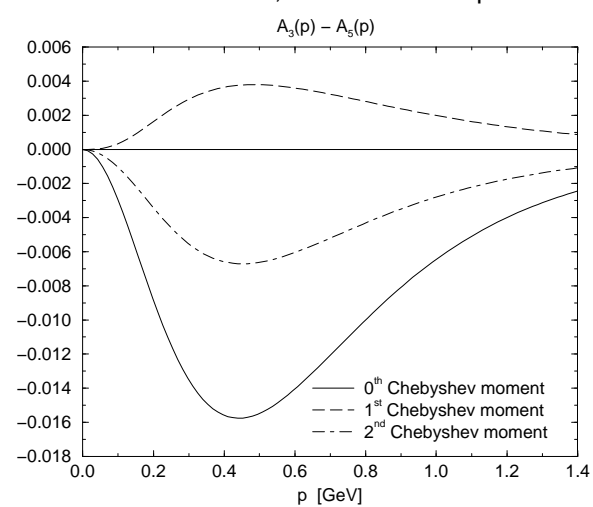

Nucleon: $l=1, s=1 / 2-$ Scalar Diquark

$\mathrm{S}_{2}(\mathrm{p})$

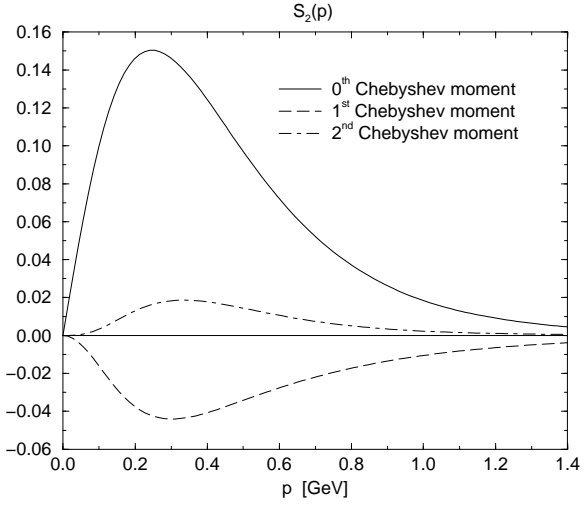

Nucleon: $\mid=1, s=1 / 2-A V$ Diquark

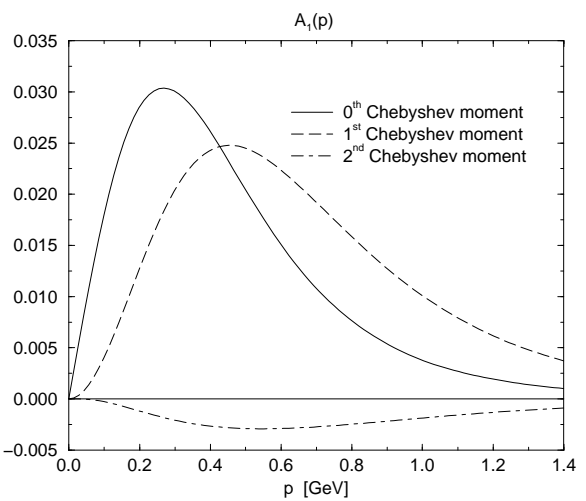

Nucleon: $I=1, s=1 / 2-A V$ Diquark $1 / 3 A_{4}(p)+2{ }_{3} A_{6}(p)$

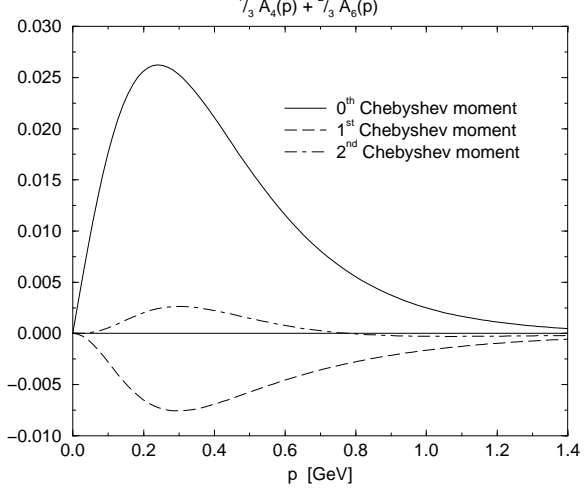

Nucleon: $I=1, s=3 / 2-A V$ Diquark

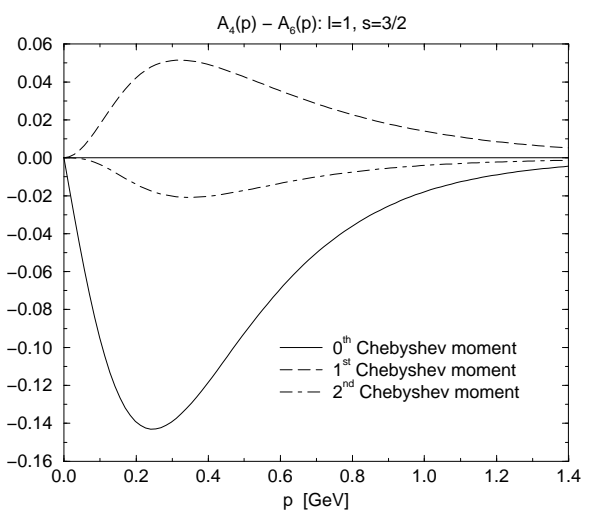

Figure 6: Scalar and axial vector amplitudes of the nucleon wave function with parameters given by $m_{u}=0.5 \mathrm{GeV}, \xi=1, \eta=0.33, d=10$ and $\Lambda=1 \mathrm{GeV}$. 
Delta: $I=0, s=3 / 2$

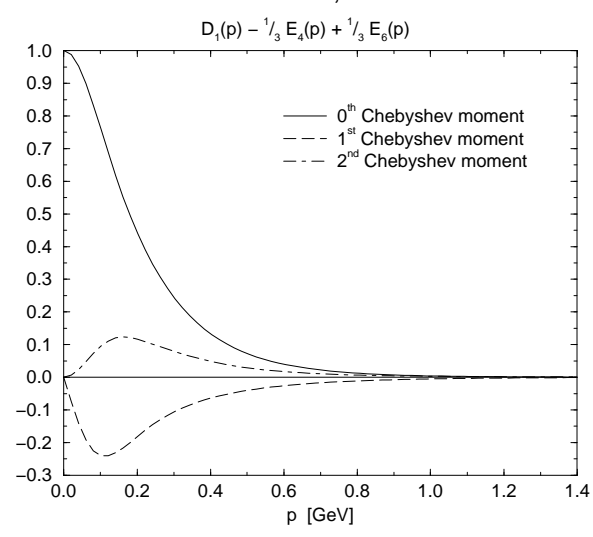

Delta: $l=1, s=3 / 2$

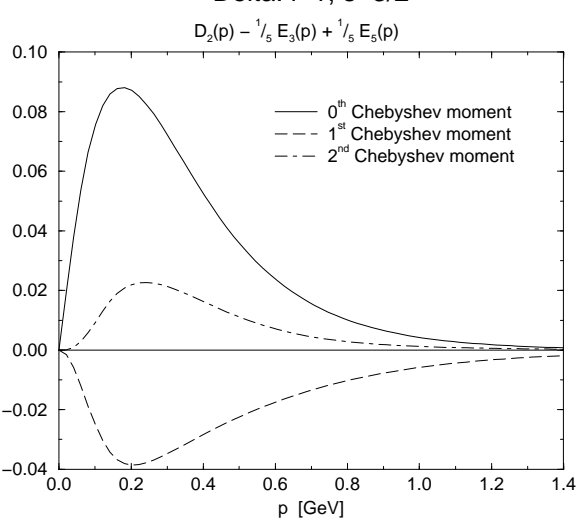

Delta: $I=2, s=1 / 2$
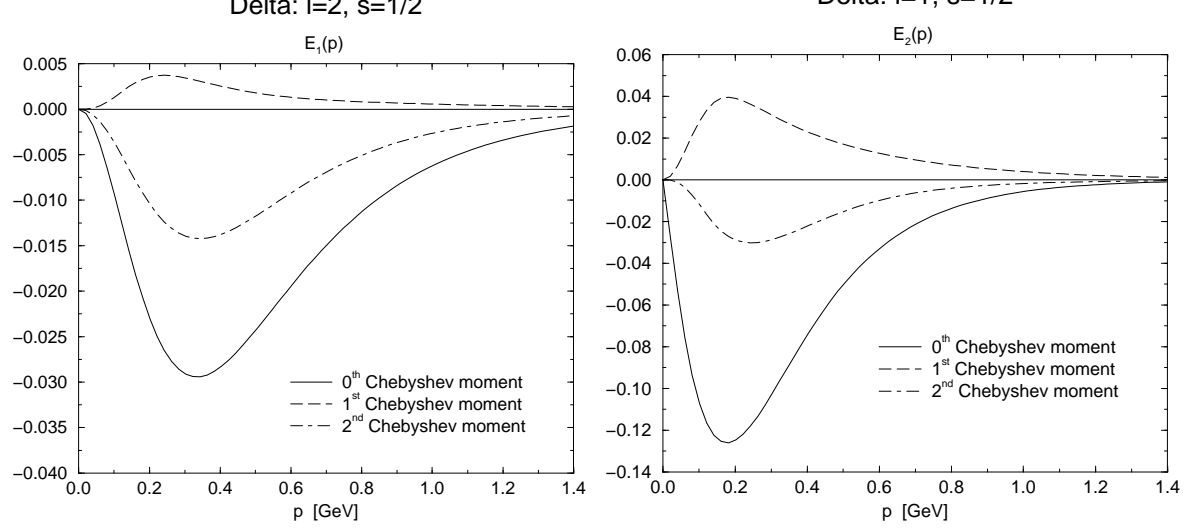

Delta: $I=2, s=1 / 2$

Delta: $l=1, s=1 / 2$

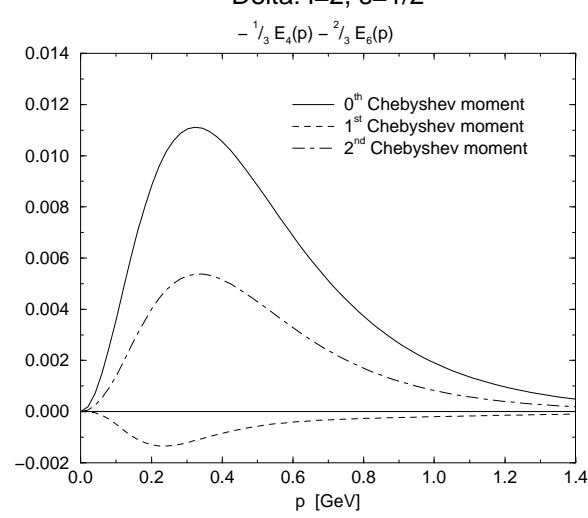

Delta: $\mid=2, s=3 / 2$
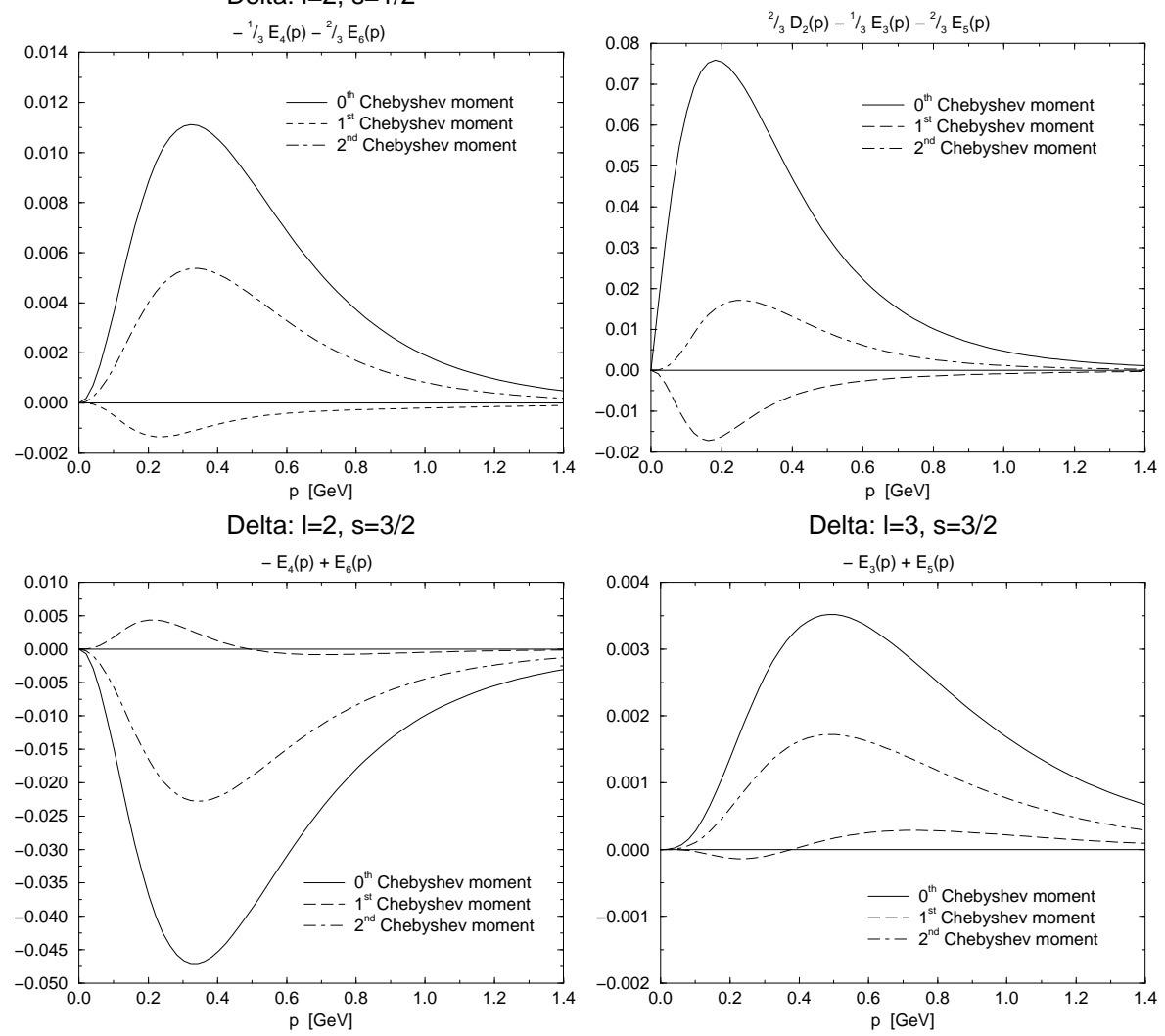

Delta: $\mid=3, s=3 / 2$

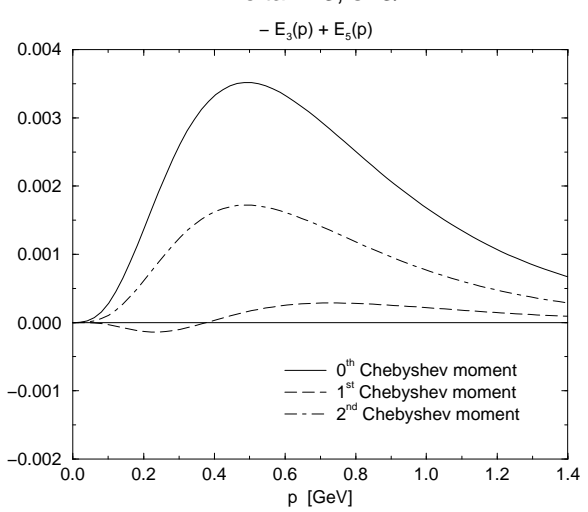

Figure 7: Axial vector amplitudes of the delta wave function with parameters given by $m_{u}=0.5$ $\mathrm{GeV}, \xi=1, \eta=0.33, d=10$ and $\Lambda=1 \mathrm{GeV}$. 


\section{Conclusions and Outlook}

In this paper we extended the covariant and confining diquark-quark model of ref. [1]] by including axialvector diquarks in the description of baryons within the Bethe-Salpeter approach. Thus we were able to calculate the octet baryons masses as well as the decuplet masses and wave functions. We implemented confinement via an effective parametrisation of the constituent propagators and demonstrated the existence of bound states beyond the pseudo-threshold.

We decomposed octet and decuplet vertex and wave functions in the Dirac and Lorentz algebra, obtaining 8 scalar functions, respectively, which we computed numerically. Two approximations to the Bethe-Salpeter equation were discussed and compared: The direct ladder approximation, and a modified ladder approximation with a special momentum routing leading to a kernel independent of the bound state mass.

In order to fix the parameters of the model preliminarily, we computed the masses of octet and decuplet for broken $S U(3)_{\text {flavour }}$ with isopin conserved. With the scalar and axialvector diquark mass assumed to be equal, the octet-decuplet mass splitting is a result of the different effects of the coupling constants in the scalar and axialvector diquark channel. For a parameter set which fits the octet and decuplet masses well, we computed vertex amplitudes and wave functions for all octet and decuplet baryons. The wave functions for baryons with different strangeness content but same spin differ mostly due to the different flavour Clebsch-Gordan coefficients, the respective scalar functions being very similar. Therefore we presented only the wave functions for the nucleon and delta out of this data. The decomposition of the wave functions in the rest frame of the bound state in terms of spin and orbital angular momentum eigenstates revealed an $s$-wave dominance in all ground state baryons stemming from both scalar and axialvector diquark contributions. The $p$-wave contributions sneaking in via the lower components of the spinors are of greater importance for decuplet baryons than for octet baryons.

Due to its special role among the other baryons, we investigated the $\Lambda$-hyperon in more detail and discussed its vertex amplitudes. In our approach, the $\Lambda$ acquires a small flavour singlet admixture which is absent in $S U(6)$ symmetric nonrelativistic quark models.

This work, together with our preceding paper [1], provides a sound basis for further applications of this approach to baryon phenomenology. The calculated amplitudes which encode the non-trivial information of a baryon as a diquark-quark bound state serve as a necessary input for the calculation of various observables. A calculation of the electromagnetic form factors of the octet and decuplet baryons is hereby the next task. Such an investigation will furthermore help to fix some of the parameters. Our aim is however to apply the covariant and confining diquark-quark model to processes, which 
are far less understood. Especially, the reactions $p \gamma \rightarrow K \Lambda$ and $p p \rightarrow p \Lambda K$, currently measured at ELSA and COSY, respectively, will serve as a stringent test of our approach. Additionally, we plan to get further insight into this picture of baryons by computing structure functions for a spectator model [30] which includes the axialvector diquark.

\section{Acknowledgement}

We thank Lorenz v. Smekal and Herbert Weigel for valuable discussions, especially the latter for insisting on his critical remarks. We are grateful to Lorenz v. Smekal for the possibility to compare with his numerical results regarding the Bethe-Salpeter eigenvalues prior to publication.

\section{A Octet and Decuplet Equations}

The symmetric and antisymmetric flavour matrices can be written down as:

$$
\begin{aligned}
t_{\mathcal{A}}^{a}= & \left\{\rho^{a=1 \ldots 3}\right\}= \\
= & \left\{\left(\begin{array}{ccc}
0 & 1 & 0 \\
-1 & 0 & 0 \\
0 & 0 & 0
\end{array}\right),\left(\begin{array}{ccc}
0 & 0 & 1 \\
0 & 0 & 0 \\
-1 & 0 & 0
\end{array}\right),\left(\begin{array}{ccc}
0 & 0 & 0 \\
0 & 0 & 1 \\
0 & -1 & 0
\end{array}\right)\right\}, \\
t_{\mathcal{S}}^{a}= & \left\{\rho^{a=4 \ldots 9}\right\}= \\
= & \left\{\left(\begin{array}{ccc}
\sqrt{2} & 0 & 0 \\
0 & 0 & 0 \\
0 & 0 & 0
\end{array}\right),\left(\begin{array}{ccc}
0 & 0 & 0 \\
0 & \sqrt{2} & 0 \\
0 & 0 & 0
\end{array}\right),\left(\begin{array}{ccc}
0 & 0 & 0 \\
0 & 0 & 0 \\
0 & 0 & \sqrt{2}
\end{array}\right),\right. \\
& \left.\left(\begin{array}{ccc}
0 & 1 & 0 \\
1 & 0 & 0 \\
0 & 0 & 0
\end{array}\right),\left(\begin{array}{ccc}
0 & 0 & 1 \\
0 & 0 & 0 \\
1 & 0 & 0
\end{array}\right),\left(\begin{array}{lll}
0 & 0 & 0 \\
0 & 0 & 1 \\
0 & 1 & 0
\end{array}\right)\right\}
\end{aligned}
$$

By these conventions flavour antisymmetric diquarks are $(u d):=u d-d u$, etc. and flavour symmetric diquarks $\sqrt{2} u u,[u d]:=u d+d u$, etc. The flavour wave functions of octet and decuplet states do not decouple once the $s$-quark breaks the symmetry. The Bethe-Salpeter equation (7) still describes nucleons (isospin is assumed to be conserved), and equation (8) still refers to $\Delta$ and $\Omega$ which possess only single-component flavour wave functions $[u u]^{\mu \rho} u$ resp. $[s s]^{\mu \rho} s$.

We use the following abbreviations to give short-hand Bethe-Salpeter equations for the remaining baryons: 


\begin{tabular}{|lll|}
\hline$(a b) c$ resp. $[a b]^{\mu} c$ & $:$ octet flavour wave functions with diquark flavour \\
& content $a b$ and spectator quark of flavour $c$ \\
{$[a b]^{\mu \rho} c$} & $:$ & decuplet flavour wave functions \\
$S_{a}$ & $:$ & quark propagator of the spectator quark $a$ \\
$D_{(a b)}$ resp. $D_{[a b]}^{\mu \mu^{\prime}}$ & $:$ & scalar resp. axialvector diquark propagator \\
$K_{a}^{\rho \lambda}=\int \frac{d^{4} p^{\prime}}{(2 \pi)^{4}} \gamma^{\rho} \tilde{S}_{a}(-q) \gamma^{\lambda}$ & $:$ & exchange kernel for quark flavour $a$, see eq. (9) for the \\
$(\rho, \lambda=1 \ldots 5)$ & definition of $\tilde{S}$ \\
\hline
\end{tabular}

Of course, different to eqs. (355), different masses corresponding to the flavour content of quark and diquark are used in the numerators and denominators of the propagators.

For octet states each flavour wave function with scalar diquarks is to be expanded in Dirac space according to eq. (17) with only the scalar amplitudes $S_{1}$ and $S_{2}$ and each one with axialvector diquarks according to the same equation with the 6 vector amplitudes $A_{1} \ldots A_{6}$. For decuplet states only flavour wave functions with axialvector diquarks are considered, they have to be expanded as indicated in eq. (24).

The Bethe-Salpeter equation for $\Xi$ baryons now reads:

$$
\begin{aligned}
\left(\begin{array}{c}
(u s) s \\
{[u s]^{\mu} s} \\
{[s s]^{\mu} u}
\end{array}\right)= & -g_{s}^{2}\left(\begin{array}{ccc}
S_{s} D_{(u s)} & 0 & 0 \\
0 & S_{s} D_{[u s]}^{\mu \mu^{\prime}} & 0 \\
0 & 0 & S_{u} D_{[s s]}^{\mu \mu^{\prime}}
\end{array}\right) \\
& \times\left(\begin{array}{ccc}
K_{u}^{5,5} & -\frac{g_{a}}{g_{s}} K_{u}^{\nu, 5} & \sqrt{2} \frac{g_{a}}{g_{s}} K_{s}^{\nu, 5} \\
-\frac{g_{a}}{g_{s}} K_{u}^{5, \mu^{\prime}} & \frac{g_{a}^{2}}{g_{s}^{2}} K_{u}^{\nu \mu^{\prime}} & \sqrt{2} \frac{g_{a}^{2}}{g_{s}^{2}} K_{s}^{\nu \mu^{\prime}} \\
\sqrt{2} \frac{g_{a}}{g_{s}} K_{s}^{5, \mu^{\prime}} & \sqrt{2} \frac{g_{a}^{2}}{g_{s}^{2}} K_{s}^{\nu \mu^{\prime}} & 0
\end{array}\right)\left(\begin{array}{c}
(u s) s \\
{[u s]^{\nu} s} \\
{[s s]^{\nu} u}
\end{array}\right)
\end{aligned}
$$

By interchanging $s \leftrightarrow u$ one obtains immediately the equation for $\Sigma$ baryons.

Broken $S U(3)$ couples the symmetric $\Lambda$ and the flavour singlet. We introduce the flavour wave functions $F_{1}=\frac{1}{\sqrt{2}}((u s) d-(d s) u), F_{2}=(u d) s$ and $\Lambda^{\mu}=\frac{1}{\sqrt{2}}\left([u s]^{\mu} d-[d s]^{\mu} u\right)$ and the equation for the physical $\Lambda$ reads:

$$
\begin{aligned}
\left(\begin{array}{c}
F_{1} \\
F_{2} \\
\Lambda^{\mu}
\end{array}\right)= & -g_{s}^{2}\left(\begin{array}{ccc}
S_{u} D_{(u s)} & 0 & 0 \\
0 & S_{s} D_{(u d)} & 0 \\
0 & 0 & S_{u} D_{[u s]}^{\mu \mu^{\prime}}
\end{array}\right) \\
& \times\left(\begin{array}{ccc}
-K_{s}^{5,5} & \sqrt{2} K_{u}^{5,5} & -\frac{g_{a}}{g_{s}} K_{s}^{\nu, 5} \\
\sqrt{2} K_{u}^{5,5} & 0 & -\sqrt{2} \frac{g_{a}}{g_{s}} K_{u}^{\nu, 5} \\
-\frac{g_{a}}{g_{s}} K_{s}^{5, \mu^{\prime}} & -\sqrt{2} \frac{g_{a}}{g_{s}} K_{u}^{5, \mu^{\prime}} & -\frac{g_{a}^{2}}{g_{s}^{2}} K_{s}^{\nu \mu^{\prime}}
\end{array}\right)\left(\begin{array}{l}
(u s) s \\
{[u s]^{\nu} s} \\
{[s s]^{\nu} u}
\end{array}\right)
\end{aligned}
$$

The $\Xi^{*}$ baryons belonging to the decuplet are described by the equation:

$$
\left(\begin{array}{l}
{[u s]^{\mu \rho} s} \\
{[s s]^{\mu \rho} u}
\end{array}\right)=-g_{a}^{2}\left(\begin{array}{cc}
S_{s} D_{[u s]}^{\mu \mu^{\prime}} & 0 \\
0 & S_{u} D_{[s s]}^{\mu \mu^{\prime}}
\end{array}\right)\left(\begin{array}{cc}
K_{u}^{\nu \mu^{\prime}} & \sqrt{2} K_{s}^{\nu \mu^{\prime}} \\
\sqrt{2} K_{s}^{\nu \mu^{\prime}} & 0
\end{array}\right)\left(\begin{array}{l}
{[u s]^{\nu \rho} s} \\
{[s s]^{\nu \rho} u}
\end{array}\right)
$$


Eigenvalue vs. Bound State Mass

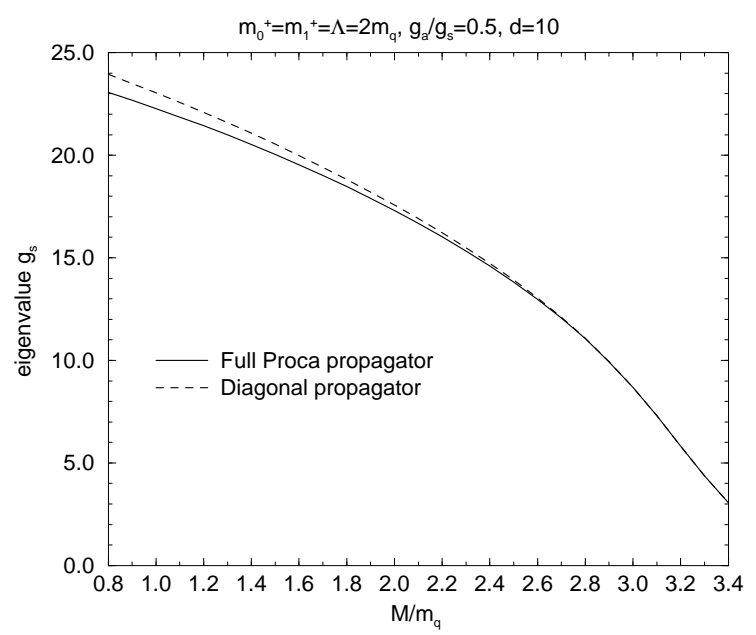

Figure 8: Eigenvalues vs. bound state mass $M$ in the octet equation. Axialvector diquark propagator with and without diagonal approximation.

As before the equation for the $\Sigma^{*}$ baryons may be obtained by interchanging $s \leftrightarrow u$. Note that we neglected contributions from a state with scalar diquark (us).

\section{B Solutions with Proca Propagator for Axialvector Diquarks}

In order to study the influence of the full Proca propagator on vertex functions of spin- $1 / 2$ baryons and the corresponding eigenvalues we are enforced to use a diquark size factor of the dipole type, writing instead of eq. (9)

$$
S(q) \rightarrow \tilde{S}(q)=S(q)\left(\frac{\Lambda^{2}}{q^{2}+\Lambda^{2}}\right)^{2} .
$$

to regularise the equation which has no stable solution otherwise.

In figure 8 we compare the eigenvalues as a function of $M$ for both the Proca propagator and its diagonal approximation. Even in regions of moderate binding the eigenvalues do not differ by more than $2 \%$ which makes the approximation of the diagonal propagator in computing the masses a reliable one. While the vertex functions are essentially the same for both choices of the axialvector diquark propagator, the $A_{1}$ components of the Bethe-Salpeter wave function differ by approximately a factor of 10 . 
Eigenvalue vs. Bound State Mass

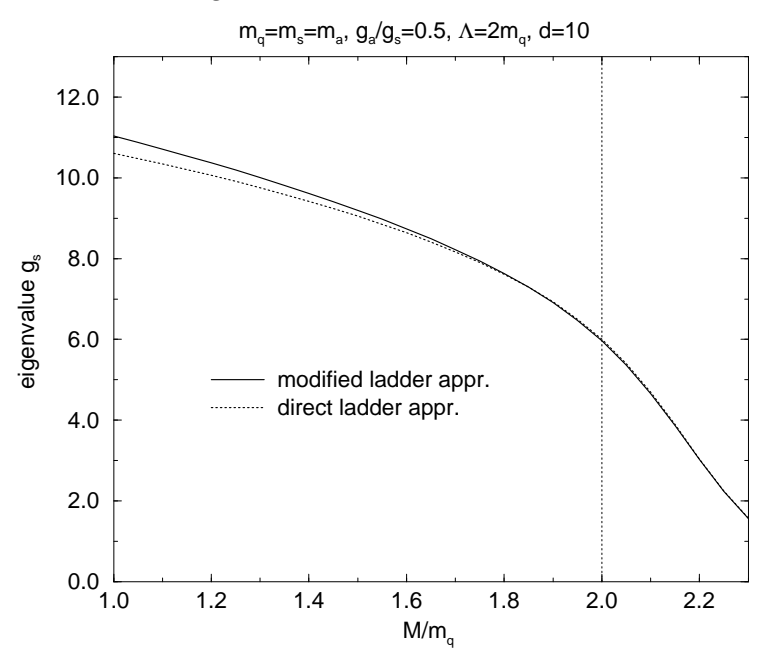

Figure 9: Eigenvalues vs. bound state mass $M$ for direct and modified ladder approximation.

\section{Direct vs. Modified Ladder Approximation}

In figure 9 we display for a representative parameter set the eigenvalues obtained in direct and modified ladder approximation. One clearly sees that for bound state masses approximately equal to the sum of the constituent masses the eigenvalues are almost identical, and even for strongly bound states the deviation is small.

However, larger deviations occur in the vertex functions. Figure 10 shows zeroth and first Chebyshev moment of $\hat{S}_{1}$ for modified and direct ladder approximation. Whereas $\hat{S}_{1}^{0}$ hardly differs for the two approaches, $\hat{S}_{1}^{1}$ receives a sign flip when switching between the two approaches. This occurs for almost all amplitudes in odd Chebyshev moments. When considering the Bethe-Salpeter equation for nucleons with $g_{a}=0$, the different sign in $\hat{S}_{1}^{1}$ causes the electric form factor of the neutron to be changed. Isospin breaking effects are also different: The neutron-proton mass difference is in the direct approach bigger than the constituent quark mass difference $m_{d}-m_{u}$ whereas in the modified ladder approximation it assumes values of approximately $0.7\left(m_{d}-m_{u}\right)$ [50]. 

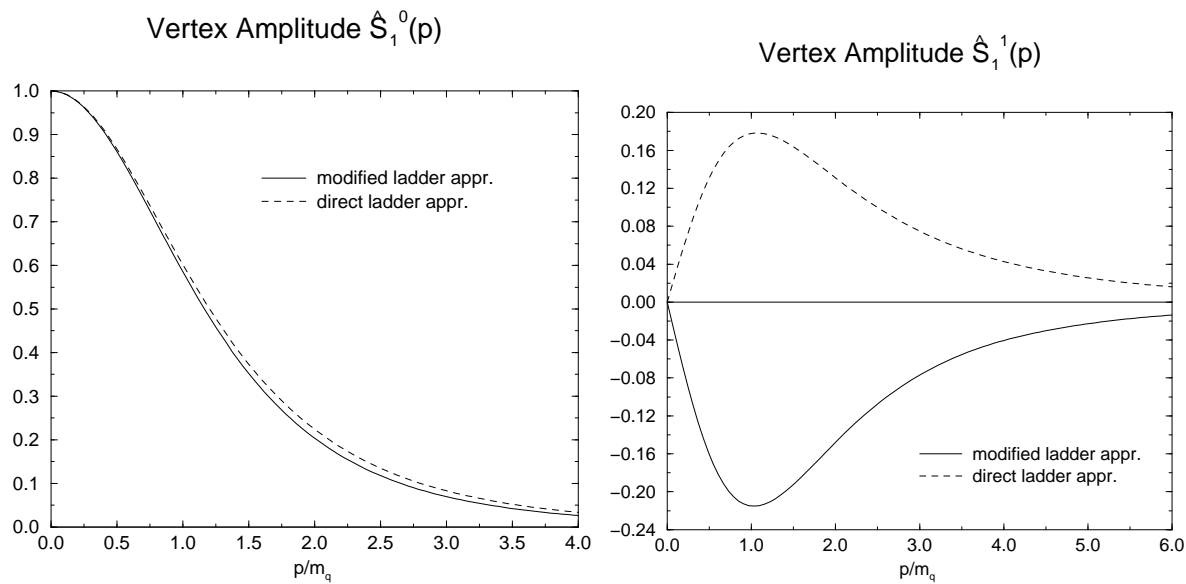

Figure 10: Zeroth (left) and first (right) Chebyshev moment of the vertex amplitude $\hat{S}_{1}$. Parameters are $m_{q}=m_{0^{+}}=m_{1^{+}}, M=1.9 m_{q}, \Lambda=2 m_{q}, g_{a} / g_{s}=0.5$ and $d=10$.

\section{References}

[1] G. Hellstern, R. Alkofer, M. Oettel and H. Reinhardt, Nucl. Phys. A627 (1997) 679.

[2] M. Anselmino, E. Predazzi, S. Ekelin, S. Fredriksson and D. B. Lichtenberg, Rev. of Mod. Phys. 65 (1993) 1199.

[3] M. Anselmino and E. Predazzi (Eds.), Proceedings of the Conference Diquark 3, Torino, Oct. 28-30,1996, World Scientific Publ. Comp., Singapore, 1997.

[4] M. Heß, F. Karsch, E. Laermann and I. Wetzorke, e-print hep-lat/9804023.

[5] T. H. R. Skyrme, Proc. Roy. Soc. A260 (1961) 127, for a recent review of the Skyrme model in flavour SU(3) see: H. Weigel, Int. J. Mod. Physics A11 (1996) 2419.

[6] G. Karl and E. Obryk, Nucl. Phys. B8 (1968) 609.

[7] D. Faiman and A. W. Hendry, Phys. Rev. 173 (1968) 1720.

[8] R. P. Feynman, M. Kislinger and F. Ravndal, Phys. Rev. D3 (1971) 2706.

[9] A. Chodos and C. Thorn, Phys. Rev. D12 (1975) 2733.

[10] P. Hasenfratz and J. Kuti, Phys. Rep. 40 (1978) 75.

[11] M. Rho, Phys. Rep. 240 (1994) 1.

[12] Y. Nambu and G. Jona-Lasinio, Phys. Rev. 122 (1961) 345.

[13] Y. Nambu and G. Jona-Lasinio, Phys. Rev. 124 (1961) 246.

[14] T. Hatsuda and T. Kunihiro, Phys. Rep. 247 (1994) 221. 
[15] D. Ebert, H. Reinhardt and M. K. Volkov, Prog. Part. Nucl. Phys. 33 (1994) 1.

[16] R. Alkofer and H. Reinhardt, Chiral Quark Dynamics, Springer-Verlag (1995).

[17] H. Reinhardt and R. Wünsch, Phys. Lett. B215 (1988) 577.

[18] R. Alkofer, H. Reinhardt and H. Weigel, Phys. Rep. 265 (1996) 139.

[19] C. V. Christov et al., Prog. Part. Nucl. Phys. 37 (1996) 1.

[20] H. Reinhardt, Phys. Lett. B244 (1990) 316.

[21] U. Vogl and W. Weise, Prog. Nucl. Part. Phys. 27 (1991) 195.

[22] A. Buck, R. Alkofer and H. Reinhardt, Phys. Lett. B286 (1992) 29.

[23] A. Buck and H. Reinhardt, Phys. Lett. B356 (1995) 168.

[24] S. Huang and J. Tjon, Phys. Rev. C49 (1994) 1702.

[25] C. Hanhart and S. Krewald, Phys. Lett. B344 (1995) 55.

[26] N. Ishii, W. Bentz and K. Yazaki, Phys. Lett. B301 (1993) 165, Phys. Lett. B318 (1993) 26, Nucl. Phys. A587 (1995) 617.

[27] U. Zückert, R. Alkofer, H. Weigel and H. Reinhardt, Phys. Rev. C55 (1997) 2030.

[28] R. T. Cahill, Nucl. Phys. A543 (1992) 63.

[29] H. Meyer, Phys. Lett. B337, 37 (1994).

[30] K. Kusaka, G. Piller, A. W. Thomas and A. G. Williams, Phys. Rev. D55 (1997) 5299.

[31] L. Faddeev, Sov. Phys. JETP 12 (1961) 1014.

[32] V. Keiner, Phys. Rev. C54 (1996) 3232, Z. Phys. A359 (1997) 91.

[33] C. D. Roberts and A. G. Williams, Prog. Part. Nucl. Phys. 33 (1994) 477.

[34] R. T. Cahill, C. D. Roberts and J. Praschifka, Phys. Rev. D36 (1987) 2804.

[35] V. Thorsson and I. Zahed, Phys. Rev. D41 (1990) 3442.

[36] C. Weiss, A. Buck, R. Alkofer and H. Reinhardt, Phys. Lett. B312 (1993) 6.

[37] H. J. Munczek and A. M. Nemirovsky, Phys. Rev. D28 (1983) 181.

[38] A. Bender, C. D. Roberts and L. v. Smekal, Phys. Lett. B380 (1996) 7.

[39] G. Hellstern, R. Alkofer and H. Reinhardt, Nucl. Phys. A625 (1997) 697. 
[40] M. Oettel and R. Alkofer, "An Iterative Solution to Fermion-Boson-Bethe-Salpeter Equations", in preparation, (1998).

[41] A. B. Henriques, B. H. Kellet and R. G. Moorhouse, Ann. of Phys. 93 (1975) 125.

[42] C. Itzykson and J. B. Zuber, Quantum Field Theory, McGraw-Hill, New York, 1985.

[43] N. Nakanishi, Prog. Theor. Phys. Suppl. 43 (1969) 1.

[44] M. A. Pichowsky and L. v. Smekal "Quark-Diquark Bound States and the Ward Identity", in preparation, (1998).

[45] W. B. Kaufmann, Phys. Rev. 187 (1969) 2051.

[46] S. Ahlig, Diploma Thesis, Tübingen University, 1998, unpublished.

[47] P. Kroll, M. Schürmann, K. Passek and W. Schweiger Phys. Rev. D55 (1997) 4315.

[48] W. Rarita and J. Schwinger, Phys. Rev. 60 (1941) 61.

[49] D. Lurié, Particles and Fields, Interscience Publishers, New York, 1968.

[50] M. Oettel, Diploma Thesis, Tübingen University, 1997, unpublished. 